\title{
ANALYZING SOLAR, COSMIC, AND GEOPHYSICAL EVENTS IN SEPTEMBER 2017, USING SHICRA SB RAS COMPLEX OBSERVATIONS
}

\section{S.A. Starodubtsev}

Yu.G. Shafer Institute of Cosmophysical Research and Aeronomy of SB RAS,

Yakutsk,Russia,starodub@ikfia.ysn.ru

D.G. Baishev

Yu.G. Shafer Institute of Cosmophysical Research and Aeronomy of SB RAS,

Yakutsk,Russia,baishev@ikfia.ysn.ru

\section{V.G. Grigoryev}

Yu.G. Shafer Institute of Cosmophysical Research and Aeronomy of SB RAS,

Yakutsk, Russia,grig@ikfia.ysn.ru

\section{R.R. Karimov}

Yu.G. Shafer Institute of Cosmophysical Research and Aeronomy of SB RAS,

Yakutsk,Russia,karimov@ikfia.ysn.ru

\section{V.I. Kozlov}

Yu.G. Shafer Institute of Cosmophysical Research and Aeronomy of SB RAS,

Yakutsk,Russia,v.kozlov@ikfia.ysn.ru

\section{A.A. Korsakov}

Yu.G. Shafer Institute of Cosmophysical Research and Aeronomy of SB RAS,

Yakutsk, Russia,korsakovaa@ikfia.ysn.ru

\section{G.A. Makarov}

Yu.G. Shafer Institute of Cosmophysical Research and Aeronomy of SB RAS,

Yakutsk,Russia,gmakarov@ikfia.ysn.ru

\author{
A.V. Moiseev \\ Yu.G. Shafer Institute of Cosmophysical Research \\ and Aeronomy of $S B R A S$, \\ Yakutsk,Russia,moiseyev@ikfia.ysn.ru
}

\begin{abstract}
We report the results of monitoring of cosmic rays and geomagnetic field along 210 magnetic meridians in Yakutia in the first half of September 2017. The energy spectrum of solar cosmic rays during Ground Level Enhancement in September 10, 2017 is estimated as $J=3027 E^{-1.99} \exp (-E / 729 \mathrm{MeV})$. We present the results of the forecast and complex analysis of the magnetic storm on September 7-9, 2017 with Dst=$124 \mathrm{nT}$. The forecast lead time is about one day. We examine how the storm affected the electric potential and VLF signal propagation from RSDN-20 radio navigation stations. Irregular $\mathrm{Pi}-\mathrm{Pi} 1$ pulsations
\end{abstract}

occurred during the September 8, 2017 magnetic storm from 12 to 20 UT. The pulsations were accompanied by variations in electrotelluric potentials and geomagnetic fields with the correlation coefficient between them $\rho(E, H)=0.5 \div 0.9$. The effects of the magnetic storm manifested themselves as an increase in the attenuation and a decrease in the phase delay of VLF radio signals.

Keywords: cosmic rays, solar flares, solar proton fluxes, magnetic storm, electric potentials, VLF radio wave propagation.

\section{GENERAL HELIO-, COSMO-, AND GEOPHYSICAL SITUATION IN SEPTEMBER 2017}

Currently, we are near the minimum of solar cycle 24 (Figure 1). During this period, it is difficult to expect any strong solar activity and corresponding space weather

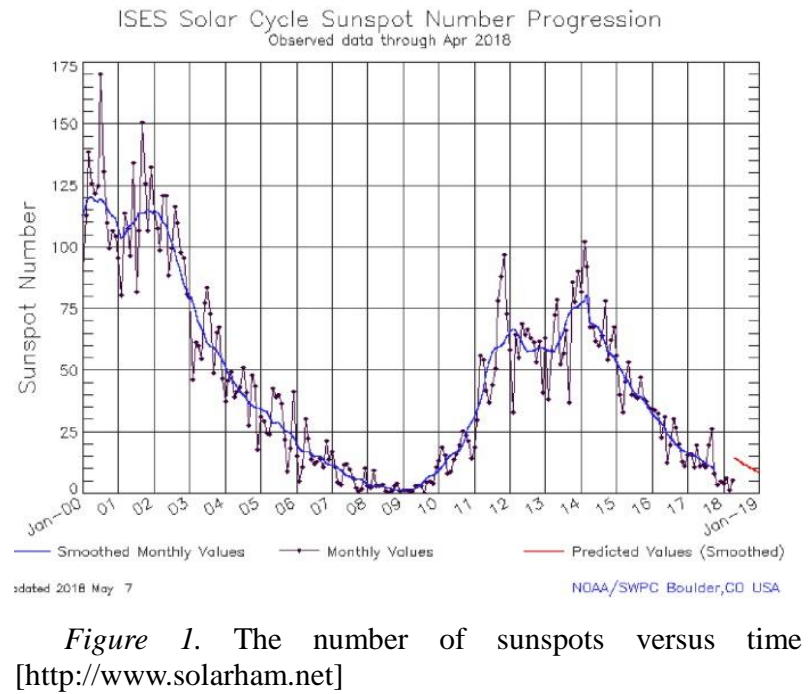

effects. However, after a long quiet period, the Sun suddenly intensified in the first half of September 2017, as manifested in its flare and coronal activity. At that time, no outstanding events occurred; nevertheless, the very high sunspot activity in the minimum of the 11-year cycle and its geophysical effects have engaged our attention. It is therefore of great interest to study them from complex observations made with different instruments of the Yu.G. Shafer Institute of Cosmophysical Research and Aeronomy of Siberian Branch of the Russian Academy of Sciences (SHICRA SB RAS), which comprise a network of stations measuring various physical parameters.

After the long quiet period, there appeared several large sunspot groups on the Sun at the beginning of September 2017 (Figure 2), which produced a number of powerful flares.

From September 4 to September 10, 2017, the Sun produced 26 class $\mathrm{M}$ and 4 class $\mathrm{X}$ flares, which was the highest manifestation of flare activity from April 2015 to May 2018. At that time, GOES spacecraft detected a number of large class $\mathrm{M}$ and $\mathrm{X}$ flares in the same active region AR 12673 (Figure 3). Information about them is available on the website [https://satdat.ngdc.noaa.gov/ sem/goes/data/new_avg/2017/09/goes15/csv/g15_xrs_1 


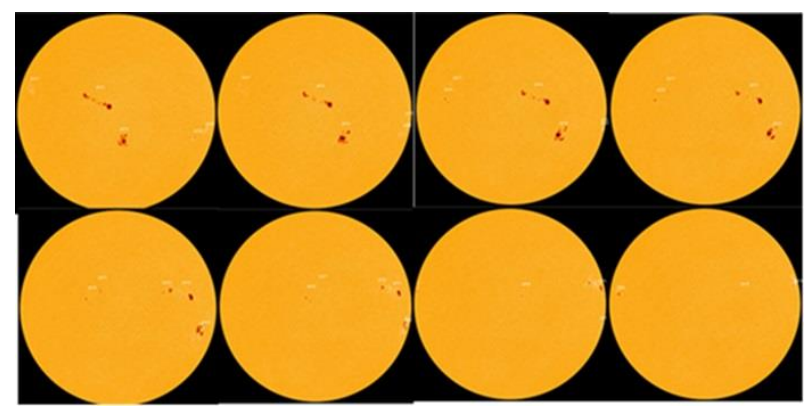

Figure 2. Passage of sunspot groups across the solar disk on September 4-11, 2017 as derived from SDO/HML data [http://www.spaceweather.com]

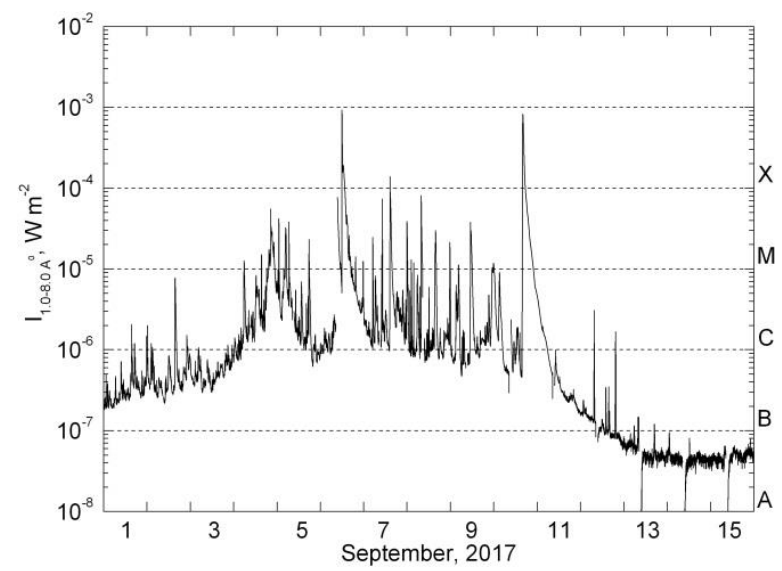

Figure 3. Solar X-ray flux in the range $1-8 \AA$ versus time in the first half of September 2017 as derived from GOES-15 observations. The right ordinate shows the widely accepted scale of X-ray flare importance corresponding to the observed $\mathrm{X}$-ray flux

m_20170901_20170930.csv]. The most powerful flares are listed in Table 1. Most of them were followed by coronal mass ejections [http://www.spaceweather.com], and in near-Earth space there were enhancements of solar cosmic ray (SCR) fluxes, Forbush decreases, and magnetic storms.

Figure 4 shows the dynamics of proton integral fluxes in six different energy channels, recorded by the GOES-15 geostationary spacecraft from September 1 to September 15, 2017 [https://satdat.ngdc.noaa.gov/ sem/goes/data/new_avg/2017/09/goes15/csv]. It can be seen that the solar particle fluxes increased in a wide energy range from 5 to $>100 \mathrm{MeV}$. Of particular interest here is the September 10 event, when the so-called Ground Level Enhancement (GLE) was detected. Notice that such events are quite rare - the ordinal number of this event is only 72 (GLE72) (recorded since 1942).

In the same period, there were geomagnetic storms. Figure 5 presents data from the Kyoto World Data Center for Geomagnetism. It illustrates the Dst dynamics in September 2017. It is seen that from September 7 to September 15, there were several geomagnetic disturbances. According to the classification ([http://en.wikipedia.org/wiki/Geomagnetic storm]), they can be classed as intense (late September 7) and moderate (early September 13 and 14) geomagnetic storms.

These unexpected effects of the high solar activity near its minimum are of great interest for fundamental and applied research into solar-terrestrial relations.
Table 1

The strongest solar flares recorded in September 2017 [http://www.solarham.net/top10.txt; https://www.spaceweatherlive.com]

\begin{tabular}{|c|c|c|c|}
\hline $\begin{array}{c}\text { Class of } \\
\text { of X-ray flare }\end{array}$ & Date & Beginning, UT & Maximum, UT \\
\hline M5.5 & $\begin{array}{c}\text { September 04, } \\
2017\end{array}$ & $20: 28$ & $20: 33$ \\
\hline X2.2 & $\begin{array}{c}\text { September 06, } \\
2017\end{array}$ & $08: 57$ & $09: 10$ \\
\hline X9.3 & $\begin{array}{c}\text { September 06, } \\
2017\end{array}$ & $11: 53$ & $12: 02$ \\
\hline M7.3 & $\begin{array}{c}\text { September 07, } \\
2017\end{array}$ & $10: 11$ & $10: 15$ \\
\hline X1.3 & $\begin{array}{c}\text { September 07, } \\
2017\end{array}$ & $14: 20$ & $14: 36$ \\
\hline M8.1 & $\begin{array}{c}\text { September 08, } \\
2017\end{array}$ & $07: 40$ & $07: 49$ \\
\hline X8.2 & $\begin{array}{c}\text { September 10, } \\
2017\end{array}$ & $15: 35$ & $16: 06$ \\
\hline
\end{tabular}

\section{COSMIC RAY INTENSITY MEASUREMENTS}

SHICRA SB RAS measures the cosmic ray (CR) intensity at two points. The first is in the Polar Geocosmophysical Observatory (PGO) in Tixie Bay $\left(71.60^{\circ} \mathrm{N}, 128.90^{\circ} \mathrm{E}\right.$, the altitude is $\left.0 \mathrm{~m}\right)$ equipped with the neutron monitor 18-NM-64, which operates in a continuous 1-min and 1-hr record mode. The second is in Yakutsk $\left(61.59^{\circ} \mathrm{N}, 129.41^{\circ} \mathrm{E}\right.$, the altitude is $\left.95 \mathrm{~m}\right)$, which is equipped with the A.I. Kuzmin CR spectrograph. It includes a complex of detectors: 1) the neutron monitor 24-NM-64; 2) four single-type muon telescopes MT with gas discharge counters SGM-14, which detect particles coming from five different directions and are set at the ground level $(0 \mathrm{~m}$ water equivalent (m w.e.)), and in insets of a special-purpose mine at 7, 20, and $40 \mathrm{~m}$ w.e.; 3 ) four new single-type scintillation muon telescopes SMT with scintillation counters ST-301, which can record particles coming from 13 different directions and are mounted at the same four levels.

The detection of muons by MT with gas discharge counters is based on triple coincidences; and by SMT,

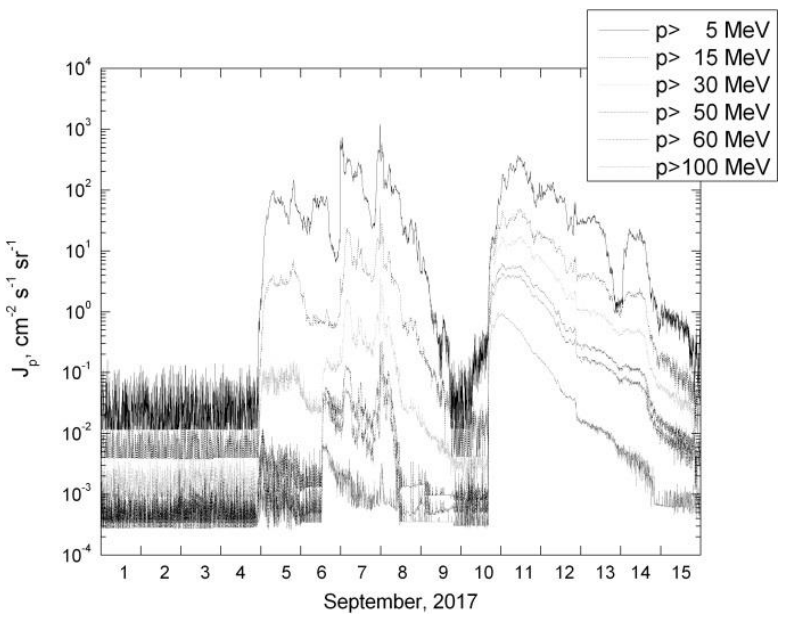

Figure 4. Integral proton fluxes of different energies recorded by GOES-15 during SCR flares in September 2017. At the top are mean energies of particles recorded by EPAD (Electron, Proton, Alpha Detector) in six different integral energy channels 


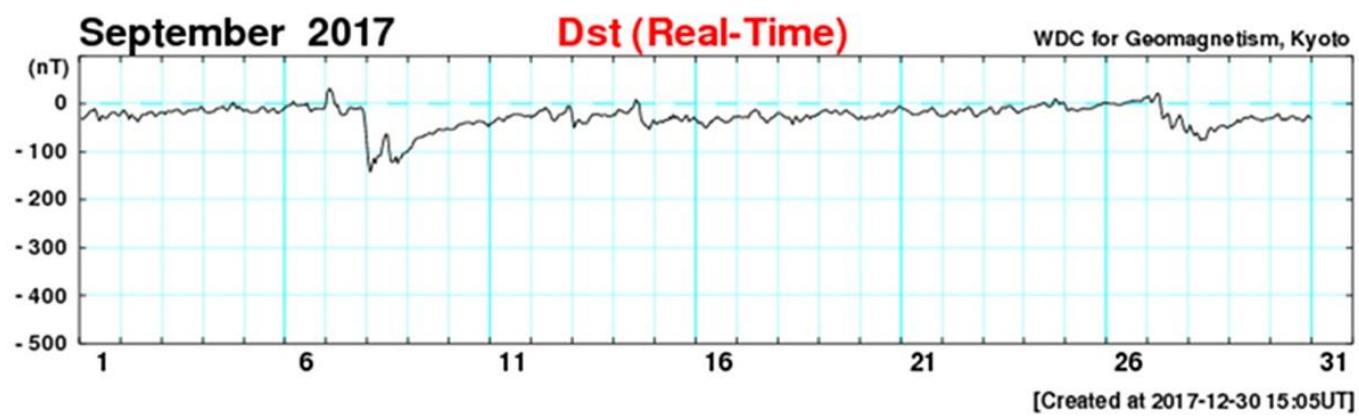

Figure 5. Dst index versus time [http://wdc.kugi.kyoto-u.ac.jp/dst_realtime/index.html]

on double coincidences [Starodubtsev et al., 2016, 2017]. For the entire complex of the detectors, we have calculated receiving characteristics (receiving vectors) accounting for their geometry, particle paths in the geomagnetic field, atmospheric effect, and spectrum of the first two angular momenta of the CR distribution function. The $\mathrm{CR}$ spectrograph operates in the continuous 1-min and 1-hr record mode. Parameters of the receiving characteristics and current data on $\mathrm{CR}$ intensity are available for all interested users [http://www.ysn.ru/ipm, http://www.ysn.ru/smt]. Information from both the $\mathrm{CR}$ stations provides a detailed insight into interplanetary medium conditions, Forbush decreases, ground level enhancements of SCR, and galactic cosmic ray (GCR) anisotropy, and allows us to solve applied research problems associated with space weather effects on Earth.

\section{GROUND LEVEL ENHANCEMENT OF COSMIC RAYS ON SEPTEMBER 10, 2017 (GLE72)}

On September 10, 2017 at 16:06 UT in the active region AR 12673, which had coordinates S08W83 by that time, an X8.2 solar flare occurred - one of the strongest in solar cycle 24 . The flare was followed by a halo coronal mass ejection, the corresponding shock wave velocity was $2900 \mathrm{~km} / \mathrm{s}$ [http://www.stce.be/ newsletter/pdf/2017/STCEnews20170915.pdf].

Approximately 20-25 min after its commencement, GOES-15 recorded an increase in the SCR flux (Figure 6).

Note that unlike some other events, such as the October 28, 2003 event (GLE65) [Krymsky et al., 2008], according to GOES-13 and -15 data there was no increase in solar particles in the low-energy spectrum region $(0.7-4 \mathrm{MeV}$ detection channel). This event was observed only at energies above 9.4 MeV [ftp://ftp.swpc.noaa.gov/pub/ lists/pchan/]. Almost at the same time, at 16:30 UT, data from neutron monitor stations Yakutsk and Tixie Bay showed a ground level enhancement of SCR (GLE72) (Figure. 7). The enhancement recorded at Yakutsk is somewhat greater than that recorded at Tixie, despite the fact that the geomagnetic cutoff threshold $R_{\mathrm{c}}$ at Yakutsk $(1.65 \mathrm{GV})$ is higher than that at Tixie Bay $(0.48 \mathrm{GV})$ (Figure 7, white line). This is due to the thick concrete ceiling in Tixie Bay station.

The use of data from the worldwide network of neutron monitor stations [http://www.nmdb.eu] along

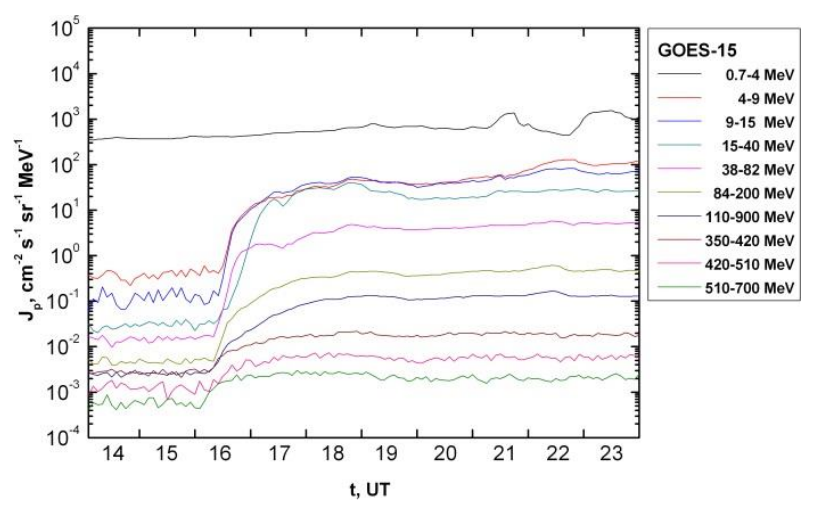

Figure 6. Differential proton fluxes of different energies recorded by GOES-15 during the September 10, 2017 SCR flare. Particle energy ranges are shown which are recorded by TELESCOPE (P1-P3), DOME (P4-P7), and HEPAD (P8-P10) in 10 different energy channels (for more details see [ftp://ftp.swpc.noaa.gov/pub/lists/pchan/README])
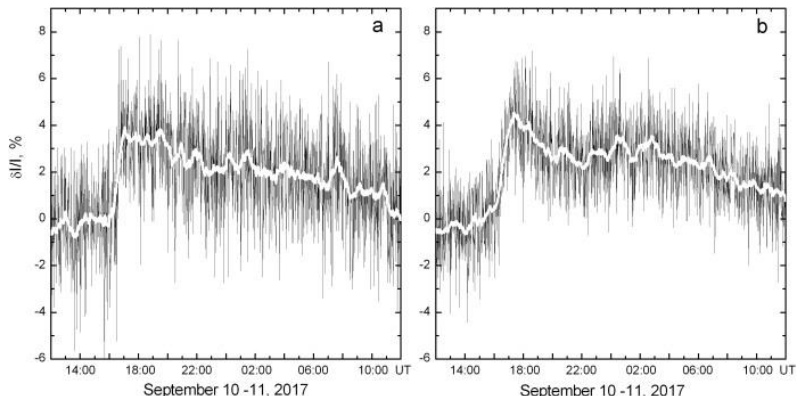

Figure 7. CR intensity vs time during GLE72 according to neutron monitor data from Tixie Bay $(a)$ and Yakutsk $(b)$. The white line indicates smoothed data

with direct measurements allowed us to determine the spectrum of the event in a wide energy range from units of $\mathrm{MeV}$ to units of $\mathrm{GeV}$ and to observe the dynamics of the SCR spectrum for this event. To do this, in addition to Yakutsk and Tixie Bay data, we used measurements made at CR stations with different geomagnetic cutoff thresholds, which detected an increase in the solar particle flux: Terra Adelie (0.01 GV), Tula $(0.30 \mathrm{GV})$, Fort Smith (0.30 GV), Apatity (0.65 GV), Oulu (0.81 $\mathrm{GV})$, Kerguelen (1.14 GV), Magadan (2.10 GV), and Irkutsk $(3.64 \mathrm{GV})$. The energy spectra of the event were calculated using the SHICRA SB RAS developed method described in detail by Krymsky et al. [2015]. 
This method enabled us to establish that in the relativistic energy region the spectrum can be described by the power function $J(E) \sim E^{-\gamma}$, where $J$ is the proton flux, $E$ is their energy, $\gamma$ is the spectrum index. In this case, at 18 and $19 \mathrm{UT}, \gamma \approx 4.5$, and at 20 and $21 \mathrm{UT}$ it becomes softer $\approx 5.0$, then it becomes more rigid again $(\approx 4.0)$. As an example, Figure 8 shows the SCR spectrum observed at 18:00 UT. Dotted straight lines are approximations of two spectral regions of the simple power function according to measurements made at GOES-15 and ground-based network of neutron monitors. It is seen that in two different energy regions the spectrum exhibits different $\gamma$ values, becoming much softer at relativistic energies. This is consistent with the results obtained by different authors (e.g., [Nymmik, 2011] and references therein). This, however, brings up the question about the particle generation mechanism capable of forming the spectrum. This question remains unanswered. On the other hand, it is known that the entire observed spectrum including direct measurements of SCR fluxes made by spacecraft is described by a more complex function $J(E)=J_{0} E^{-\gamma} \exp \left(E / E_{0}\right)$ [Ellison, Ramaty, 1985; Lovell et. al., 1998]. This spectrum is naturally set when particles are accelerated by shock fronts in the lower solar corona. The respective quasi-linear theory of regular SCR acceleration has been elaborated and described in detail in [Berezhko, Taneev, 2013].

Indeed, the entire SCR energy spectrum observed on September 10 at 18:00 UT can be satisfactorily described by the approximating function $J=3027 E^{-1.99} \exp (-E / 729$ $\mathrm{MeV}$ ) (Figure 8). Given the above fact of recording of fast CME during the flare, we can assume that in this case the SCR generation is due to particle acceleration by the coronal shock front. This can, however, be confirmed only by corresponding model calculations and their comparison [Berezhko, Taneev, 2013; Krymsky et al., 2015].

Note also that, despite the lower geomagnetic cutoff threshold at Yakutsk compared to other stations, which

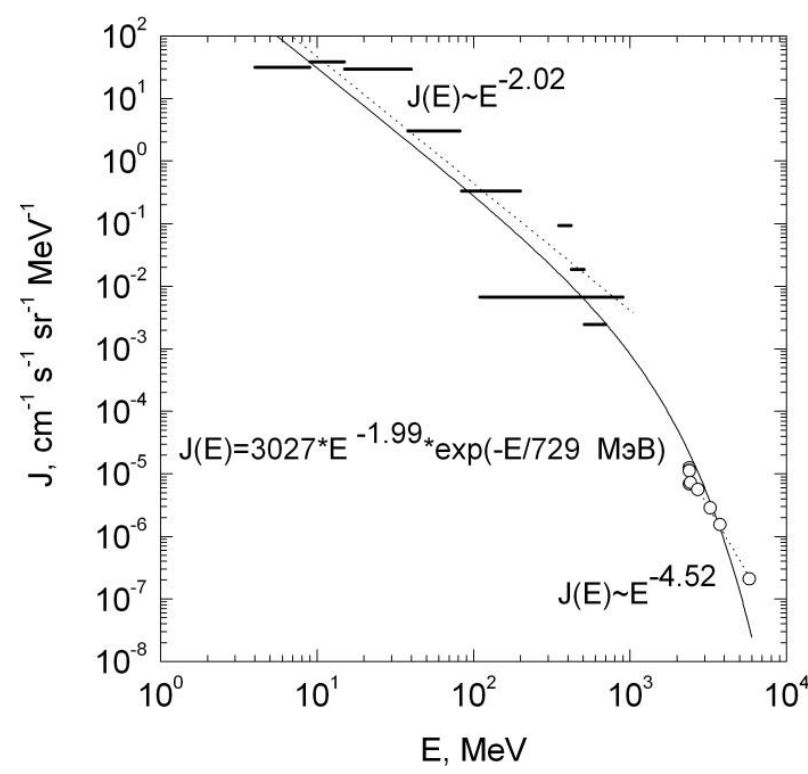

Figure 8. SCR flux on September 10, 2017 at 18:00 UT vs particle energy during GLE72. Horizontal lines mark nine differential channels of GOES-15 proton flux measurements; open circles indicate neutron monitor measurements recorded the solar particle flux enhancement, muon telescopes of the CR spectrograph did not detect this event even at $0 \mathrm{~m}$ w.e. This circumstance is due to the fact that the maximum of coupling coefficients for the vertical direction falls at high energies $(>10 \mathrm{GeV})$, and for 2-3 GeV their values decrease by more than two orders of magnitude [Starodubtsev et al., 2016].

\section{FORECAST FOR THE SEPTEMBER 7, 2017 GEOMAGNETIC STORM}

To forecast geomagnetic activity, SHICRA SB RAS has been monitoring space weather by measuring the CR intensity since 2013. The forecast is made in real time with the use of 1-hr measurements from the worldwide network of neutron monitors [http://www.nmdb.eu]. It is based on calculations of 3D GCR anisotropy made with the global survey method [Grigoryev, Starodubtsev, 2015; Grigoryev et al., 2017; Grigoryev et al., 2013].

Figure 9 shows the results of the forecast for the September 7, 2017 geomagnetic storm. This Figure is drawn by automatic calculation of CR anisotropy parameters at 08:32 of local Yakutsk time on September 7, which corresponds to 23:32 UT on September 6, 2017. From the characteristic behavior of the diurnal CR anisotropy described in [Grigoryev, Starodubtsev, 2015; Grigoryev et al., 2013], on September 6 we forecasted the expected onset of the geomagnetic storm. The forecast is made automatically if amplitudes are $A_{x}>0.5 \%$ and $A_{z}>0.5 \%$ or $A_{y}>0.5 \%$ and $A_{z}>0.5 \%$. This is shown by the caption at the bottom, which exists on the screen for only a day, and then disappears. Indeed, the worldwide network of magnetometers recorded an intense magnetic storm (Figure 5) and a Forbush decrease in CR intensity (Figure 10) at the end of September 7, 2017.

Note that the probability of the forecast for geomagnetic storms with $D s t<-50 \mathrm{nT}$ is about $70 \%$ and its current results are available at [http://www.ysn.ru/ starodub/SpaceWeather/global_sur vey_real_time.html].

\section{GEOMAGNETIC FIELD MEASUREMENTS}

The SHICRA SB RAS meridional network consists of six observation points in Yakutia; it is unique in Russia and provides magnetic observations of auroral and sub-auroral zones in latitudinal and longitudinal sections.

In the area of research in geomagnetic field and magnetic disturbances, SHICRA SB RAS participates actively in international projects INTERMAGNET [http://www.intermagnet.org] and MAGDAS [http://magdas2.serc.kyushu-u.ac.jp/station/index.html].

Under the international project MAGDAS, the fluxgate magnetometers MAGDAS-9 are located along the $190^{\circ}-210^{\circ}$ magnetic meridians on Is. Kotelny $\left(76^{\circ} 00^{\prime}\right.$ $\left.\mathrm{N}, 137^{\circ} 54^{\prime} \mathrm{E}\right)$, in PGO Tixie Bay $\left(71^{\circ} 36^{\prime} \mathrm{N}, 128^{\circ} 47^{\prime} \mathrm{E}\right)$, in Chokurdakh $\left(70^{\circ} 37^{\prime} \mathrm{N}, 147^{\circ} 55^{\prime \prime} \mathrm{E}\right)$, Zyryanka $\left(65^{\circ} 44^{\prime}\right.$

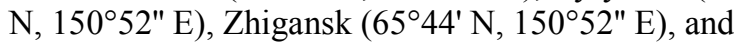




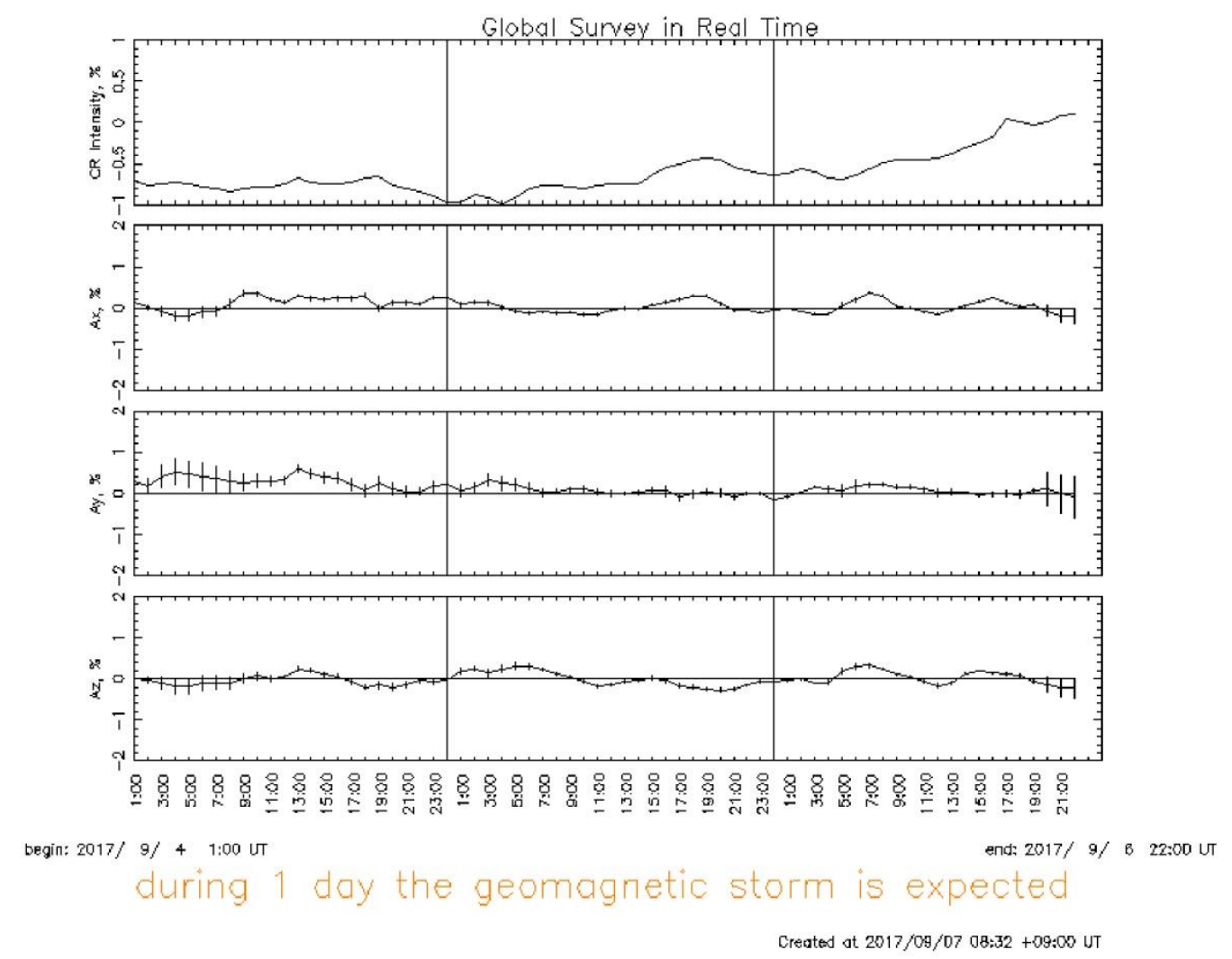

Figure 9. Isotropic intensity and CR anisotropic components (in GSE coordinate system) vs time, calculated by the global survey method from 01:00 UT on September 4 to 22:00 UT on September 6, 2017

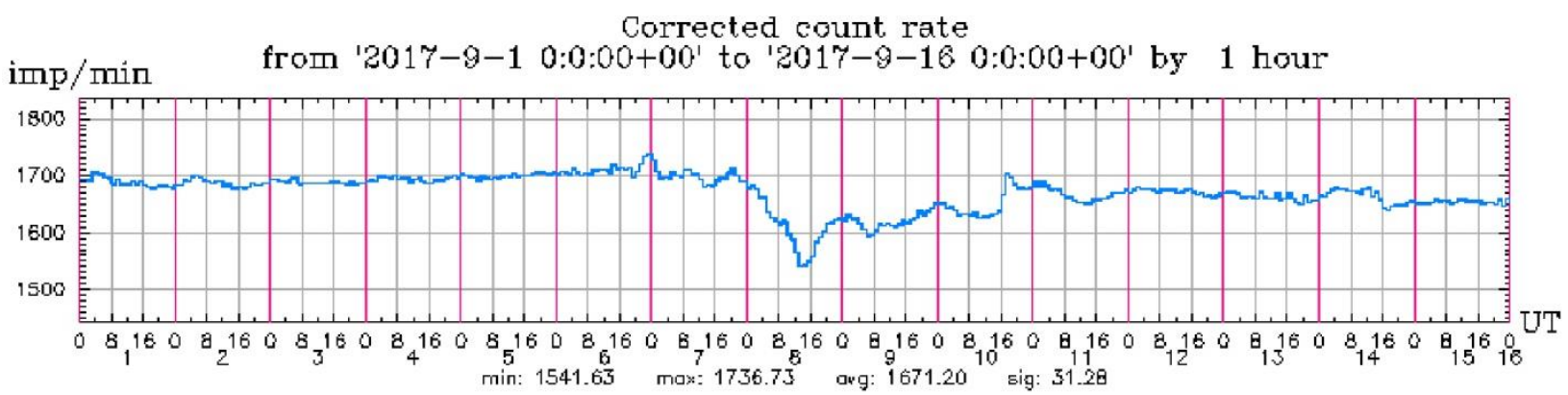

Figure 10. The CR intensity corrected for pressure vs time as derived from neutron monitor measurements at Yakutsk station in September 2017. The numbers at the bottom represent the minimum and maximum CR intensity, its average value, and standard deviation for the given period, which are calculated using aggregate functions of the PostgreSQL database from 1-min data (the Figure is the result of the interactive query at [http://www.ysn.ru/ipm])

Yakutsk (615' N, 129³9' E) [Baishev et al., 2013]. Under the project INTERMAGNET, geomagnetic field measurements are carried out only at Yakutsk observatory with the digital magnetometer system INTERMAGNET [Moiseev et al., 2011].

\section{MAGNETIC FIELD MEASUREMENTS IN YAKUTSK}

Figure 11 presents the observations of the magnetic field full vector $\mathbf{F}$ and its projections at Yakutsk magnetic observatory for September 1-15, 2017. The projection of this vector on the $\mathrm{X}$-axis $\left(H_{x}\right)$ is called the north component with direction to the north geographic pole; on the Y-axis $\left(H_{y}\right)$, the east component; and on the $\mathrm{Z}$-axis $\left(H_{z}\right)$, the vertical component. The measurements were performed using a 3-component fluxgate magnetometer (FGE DMI, Denmark). Figure 11 suggests that in this period in Yakutsk there were three magnetic disturbances of different intensities recorded on September 7, 8, and 14. The previously mentioned magnetic storm of September 13 was not observed in Yakutsk. The September 7 and 8 magnetic storms are likely, however, to be a manifestation of one intense storm having a complex structure (Figure 5).

Variations in the spectra of geomagnetic field fluctuations in data from both ground observatories and geostationary spacecraft before solar proton events have been repeatedly observed by many investigators [Cheng, 1991; Kobrin et al., 1985].

Of particular interest, therefore, is to monitor the dynamics of the spectra of fluctuations of the magnetic 

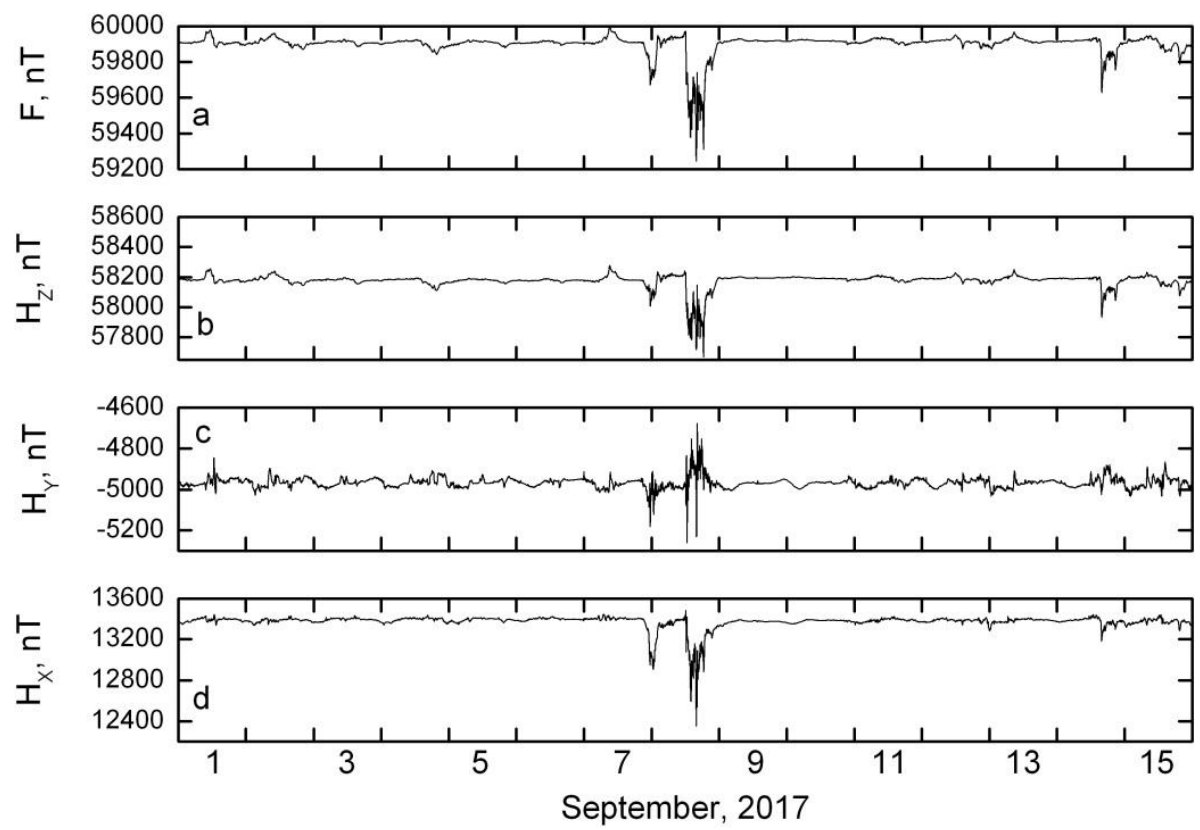

Figure 11. Total magnetic field vector $\mathbf{F}(a)$ and its projections $H_{z}, H_{y}$, and $H_{x}(b-d)$ vs time in early September 2017 as derived from measurements at Yakutsk magnetic observatory

field full vector F in Yakutsk during the September 2017 events. This is all the more important that geomagnetic conditions are determined by space weather, studies of which are currently relevant. To analyze in more detail and find possible causes of the expected dynamic changes in the spectra of magnetic field fluctuations, in addition to the 1-min measurements from Yakutsk magnetic observatory we have used 1-min measurements of the magnetic field and solar wind (SW) plasma parameters from the known database OMNI [https://cdaweb.sci.gsfc.nasa.gov/index.html]. It should be noted that the detached terrestrial shock wave has already been shifted to the bow, and therefore there is nothing to do but take into account the disturbance propagation time from this point to the point of magnetic field detection, which is a small quantity of order of $1 \mathrm{~min}$. When calculating the spectral characteristics, the length of time series was taken equal to 1 day, and the fluctuation spectra themselves were determined using the standard Blackman-Tukey method with the Tukey correlation window [Blackman, 1958].

As an example, Figure 12 presents power spectra of fluctuations of the full vector $\mathbf{F}$ of Earth's magnetospheric magnetic field, measured at Yakutsk magnetic observatory, and interplanetary magnetic field modulus $B$ for individual time intervals on September 3, 6,8 , and 15,2017 . The first interval corresponds to the geomagnetically quiet period; the second, to the period preceding the disturbance; the third, to the magnetic storm; the fourth, to the period following the slight and short geomagnetic disturbance in Yakutsk. In the analysis of the magnetic field fluctuations, the following has engaged our attention.

1. The spectra are fairly dynamic - their power varies by orders of magnitude depending on the observation period.

2. The spectra of geomagnetic field fluctuations are
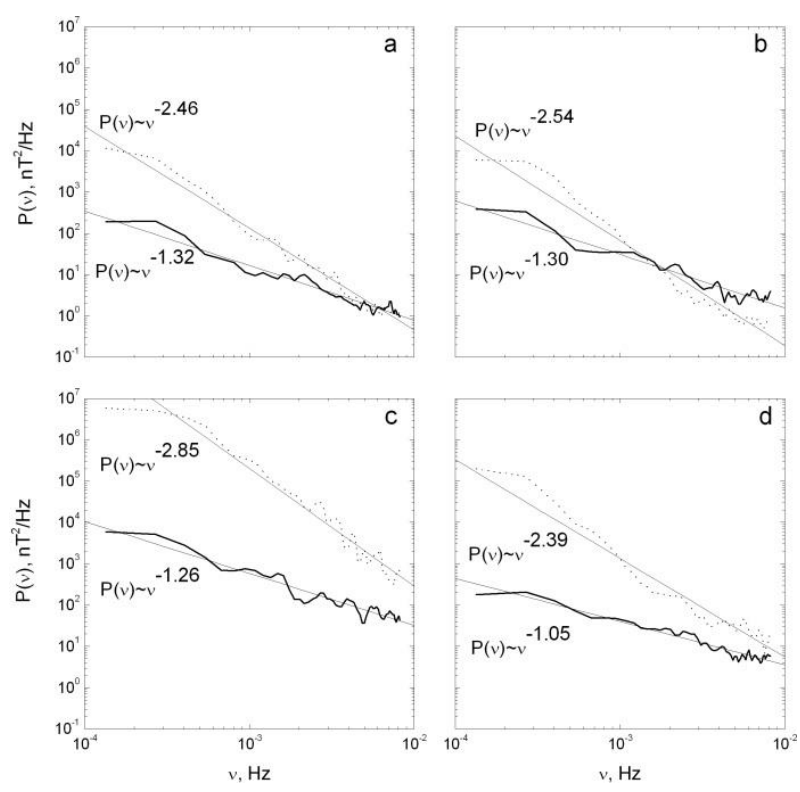

Figure 12. Power of magnetic field strength full vector fluctuations $\mathbf{F}$ vs frequency as derived from measurements of Yakutsk magnetic observatory (dashed curve), and interplanetary magnetic field modulus $B$ according to measurements made using the OMNI database for September $3(a), 6(b), 8(c)$, and $15(d), 2017$. Solid lines indicate an approximation of the fluctuation spectra by a power function

softer - their index is at least by 1 more than the corresponding index of the interplanetary magnetic field.

3. The low-frequency region of the spectra is most variable - the power of fluctuations in it can vary by several orders of magnitude, whereas in the highfrequency part, it hardly varies by one order of magnitude.

A more detailed analysis of daily spectra of the fluctuations for the entire time period under study shows 
that their most pronounced variations occur against dynamic changes in SCR fluxes and SW parameters. Note, however, that not all interplanetary disturbances affect the geomagnetic field - such as geomagnetic storms (Figure 13). To understand this, we should take into account both the geometry at which Earth crosses different large-scale SW disturbances and the level of MHD turbulence in their vicinity [Plotnikov et al., 2014; Shadrina, Starodubtsev, 2016a, b; Shadrina et al., 2012, 2014]. To establish the nature of the spectra of geomagnetic field fluctuations requires, however, further systematic research during observation of other magnetic storms with the use of direct measurements of medium parameters made by spacecraft and geostationary satellites. Thus, from the joint analysis of Figures 11-13 we can conclude that the dynamic changes in the spectra of magnetic field fluctuations $\mathbf{F}$ are mainly determined by the state of the interplanetary medium (space weather) in Earth's orbit.

\section{ANALYSIS OF THE SEPTEMBER 7- 8, 2017 EVENT}

Of particular interest is the more detailed study of geomagnetic measurements on the Yakutsk meridian on September 7 and 8, 2017, when an intense geomagnetic storm occurred (Figure 5). For this purpose, we have used 1-min measurements made at Yakutsk (corrected geomagnetic latitude $\Phi^{\prime}=56.3^{\circ} \mathrm{N}$ and longitude $\left.\Lambda^{\prime}=201.0^{\circ} \mathrm{E}\right)$, Zhigansk $\left(\Phi^{\prime}=61.4^{\circ} \mathrm{N}\right.$, $\left.\Lambda^{\prime}=194.4^{\circ} \mathrm{E}\right)$, and Tixie Bay $\left(\Phi^{\prime}=66.0^{\circ} \mathrm{N}, \Lambda^{\prime}=197.5^{\circ}\right.$ E). Figures 14, 15 show the horizontal $H$ and vertical $Z$ geomagnetic field components measured at these stations on September 7 and 8, 2017, if in UT. The measurements are made by MAGDAS-9 threecomponent flux-gate magnetometers. Figure 14 suggests that the maximum positive and negative deviations of the $H$ geomagnetic field component in Tixie Bay exceeded the absolute value of $500 \mathrm{nT}$, whereas in Zhigansk, 400 and $900 \mathrm{nT}$. The analysis of Figure 14 leads to the conclusion that on September 7, 2017 an intensive equivalent 2-vortex current system
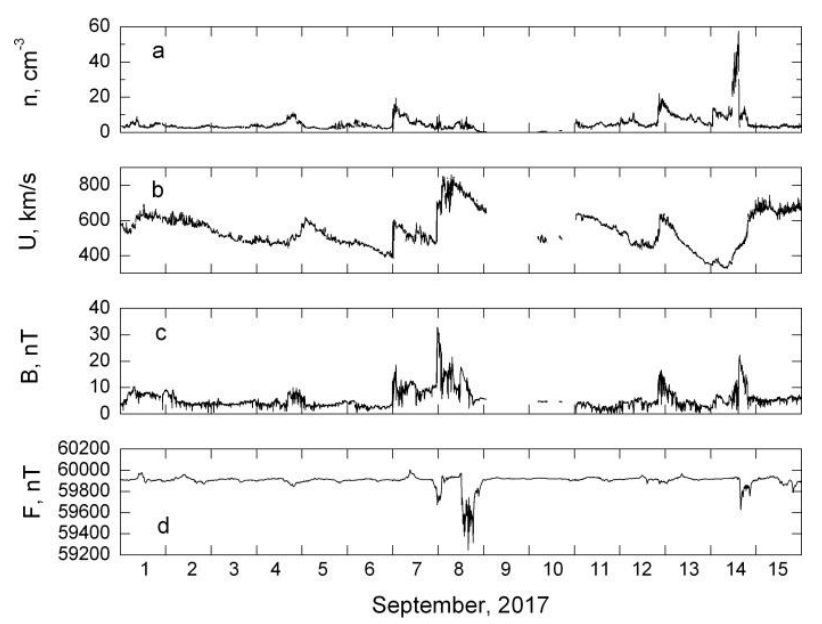

Figure 13. Density $n(a)$ and velocity $U(b)$ of the solar wind, interplanetary magnetic field modulus $B(c)$, as well as the full vector of the magnetic field strength $\mathbf{F}(d)$ in Yakutsk vs time in the first half of September 2017

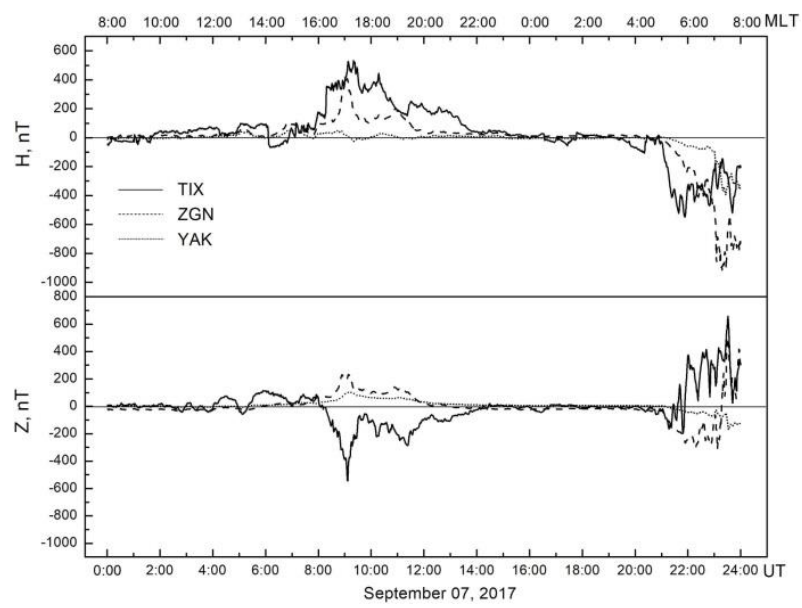

Figure 14. Variations in the horizontal $(H)$ and vertical $(Z)$ geomagnetic field components in Yakutsk, Zhigansk, and Tixie Bay on September 7, 2017

developed with the auroral eastward electrojet in the dusk sector (16:00-22:00 MLT) and the westward electrojet in the late dawn sector (05:00-08:00 MLT). The variations in the $H$ and $Z$ field components indicate that the eastward and westward electrojets were located between Tixie Bay and Zhigansk at latitudes approximately between $\Phi^{\prime} \approx 62-64^{\circ} \mathrm{N}$.

The variations in the $H$ and $Z$ geomagnetic field components at the three stations on September 8, 2017 (Figure 15) represent the effects of westward currents in the pre-noon sector (08:00-10:45 MLT) (continuation of the westward electrojet of the previous day) and in the midnight and early dawn sector (20:00-06:00 MLT). It is likely that the electrojet recorded in the second sector is strengthened and moved toward midnight hours, as well as to the south by the westward electrojet of the preceding day. It is seen that the maximum negative deviations of the $H$ component in the pre-noon sector in Tixie Bay were as great as $600 \mathrm{nT}$; and in Zhigansk, $1200 \mathrm{nT}$; whereas in the midnight and in the early dawn sector in Tixie Bay they exceeded $850 \mathrm{nT}$; and in Zhigansk, $1350 \mathrm{nT}$. The variations in $H$ and $Z$ geomagnetic field components suggest that the electrojet was located to the south of Zhigansk but to the north of Yakutsk approximately at $\Phi^{\prime} \approx 59^{\circ} \mathrm{N}$

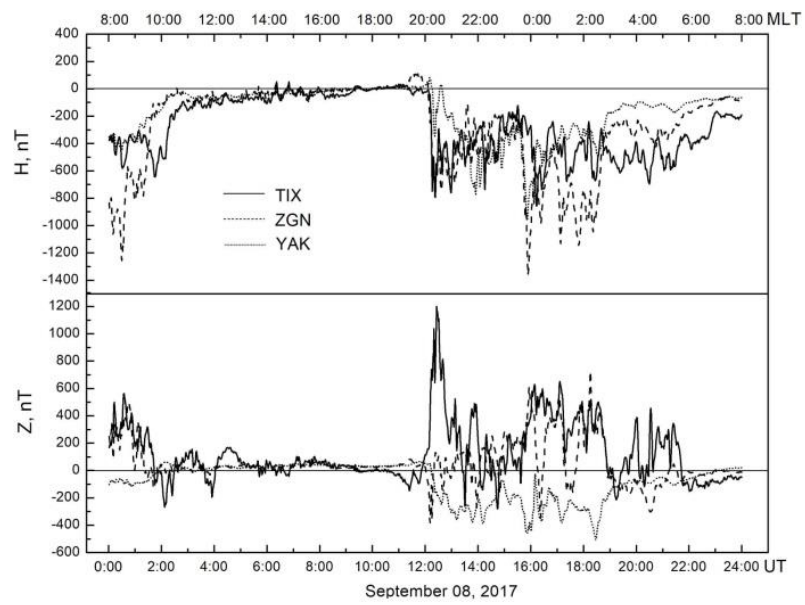

Figure 15. The same for September 8, 2017 


\section{ELECTRICAL POTENTIAL MEASUREMENTS}

The Oybenkel radiophysical station of SHICRA SB RAS $\left(61^{\circ} 55^{\prime} \mathrm{N}, 129^{\circ} 21^{\prime} \mathrm{E}\right)$, located a few tens of kilometers from Yakutsk far from man-made statics, has been measuring electric potentials since 2017. Electrotelluric measurements are made according to the standard scheme [Krolevets, Kopylova, 2003], which involves recording the potential difference between two pairs of electrodes dug into the ground, the so-called measuring lines, oriented north-south $(\mathrm{N}-\mathrm{S})$ and eastwest $(\mathrm{E}-\mathrm{W})$. Two pairs of measuring lines $100 \mathrm{~m}$ long each are oriented in these directions. Then, these measurements are used to calculate the electrotelluric field strength $E$.

\section{VARIATIONS IN NATURAL ELECTRIC POTENTIALS IN YAKUTSK AND THEIR RELATIONSHIP WITH MAGNETIC FIELD DISTURBANCES}

Of particular interest is a comparative analysis of parameters of magnetic field components and electric potentials. Consider in more detail the behavior of natural potentials during the development of the ring current (RC) on September 8, 2017.

Variations of 1-hr Dst index during the storm are shown in Figure 16. The highest magnetic disturbances in this storm $D s t=-142 \mathrm{nT}$ were observed at 02 UT (the first RC amplification) and at 14-18 UT when Dst varied within $-120 \div-142 \mathrm{nT}$ (the second RC amplification). From 12 to 19 UT, we observed $E$ variations up to $40 \mathrm{mV} / 100 \mathrm{~m}$. The variations in geomagnetic fluctuations in $X$ and $Y$ components measured with the geophysical instrument ADU-07 mounted at the Oybenkel radiophysical station, and the variations in natural potentials $E$ measured with the same instrument at a distance of $100 \mathrm{~m}$ away are depicted in Figure 17.

The designated period of the existence of large pulsations both in magnetic components and in natural electric potentials is shown in Figures 18, 19.

During the September 8, 2017 magnetic storm from 12 to 20 UT (Yakutsk nighttime), there were irregular pulsations in a wide range of periods - from Pi3 $(T>150 \mathrm{~s})$ to Pi1 ( $T=1 \div 40 \mathrm{~s}$ ). Spectrograms of the variations in the

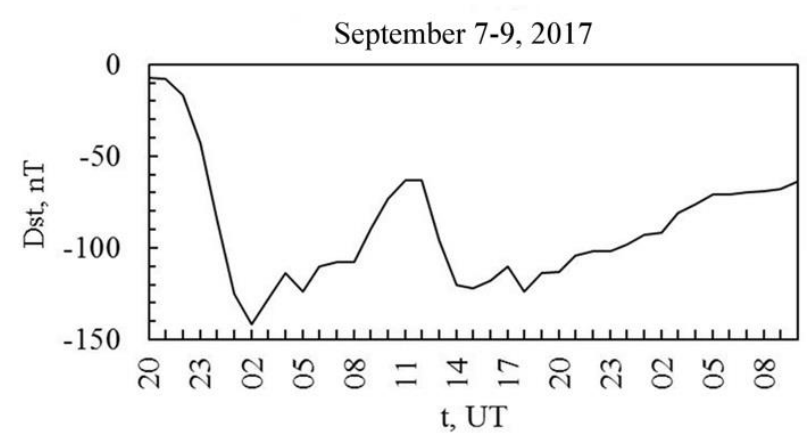

Figure 16. Variations in Dst during the severe magnetic storm of September 7-8, 2017 magnetic components during the RC amplifications on September 8, 2017 are presented in Figure 19.

Spectrograms of variations in $\mathrm{N}-\mathrm{S}$ and $\mathrm{E}-\mathrm{W}$ vector components $\mathbf{E}$ during the second RC amplification on September 8, 2017 are presented in Figure 20. Note that the $\mathbf{E}$ variations, constructed from the $\mathrm{N}-\mathrm{S}$ and $\mathrm{E}-\mathrm{W}$ components, particularly well exhibit irregular pulsations with varying upper frequency of the range, typical of irregular Pil pulsations [Kleymenova, 2007].

The correlation coefficient between the natural potential difference $E_{\mathrm{N}-\mathrm{S}}, E_{\mathrm{E}-\mathrm{w}}$ and respective $X$ and $Y$ magnetic components during the pulsations lasting 8.33 hrs in the time interval 12-20 UT during the second RC amplification on September 8, 2017 is $\rho\left(E_{\mathrm{N}-\mathrm{S}}\right.$, $\mathrm{Y})=0.77 \pm 0.1$ at a significance level $\alpha<0.005$ and $\rho\left(E_{\mathrm{E}-\mathrm{w}}\right.$, $X)=0.65 \pm 0.1 \quad(\alpha<0.005)$. The range of variation $\rho\left(E_{\mathrm{N}-\mathrm{S}}\right.$, $Y)=0.5 \div 0.9$ and $\rho\left(E_{\mathrm{E}-\mathrm{w}}, X\right)=0.52 \div 0.9$. According to literature data, the correlation coefficient between geomagnetic field variations and telluric currents is 0.833 [Aleksandrov et al., 1972]. In the afternoon, average amplitudes of natural potentials $E$ and magnetic field micropulsations $H$ within $0-500 \mathrm{nT}$ are related by $E(\mathrm{mV} / \mathrm{km}) \sim H(\mathrm{nT})$ [Hessler, Wescott, 1959].

Thus, we can conclude that the Oybenkel radiophysical station during the September 8, 2017 magnetic storm from 12 to 20 UT, Yakutsk nighttime, observed irregular pulsations in a wide range of periods - from Pi3 to Pi1. They occurred with variations in natural potentials of electrotelluric and geomagnetic fields with the correlation coefficient between them $\rho(E, H)=0.5 \div 0.9$ at the significance level $\alpha<0.005$.

\section{DETECTION OF RADIO NOISE AND SIGNALS FROM VLF RADIO STATIONS}

Since 2009, SHICRA SB RAS has been recording signals from the radio navigation system RSDN-20 [Karimov et al., 2012]. Transmitters are located near Novosibirsk ( $\left.55^{\circ} 45^{\prime} \mathrm{N}, 82^{\circ} 27^{\prime} \mathrm{E}\right)$, Krasnodar $\left(45^{\circ} 24^{\prime} \mathrm{N}\right.$, $\left.38^{\circ} 9^{\prime} \mathrm{E}\right)$, and Khabarovsk $\left(50^{\circ} 4^{\prime} \mathrm{N}, 136^{\circ} 36^{\prime}\right.$ E) and emit VLF radio pulses $(v=3 \div 30 \mathrm{kHz})$. We record RSDN-20 signals at frequencies of 11.904, 12.649, and 14.881 $\mathrm{kHz}$. At these frequencies during the pause between radio pulses of the radio stations, we also record the radio noise intensity. A radio signal is received by an electrical whip antenna (an effective height of $2 \mathrm{~m}$ ), and then, after pre-amplification (a gain of $32 \mathrm{~dB}$ in the 0.3$100 \mathrm{kHz}$ frequency band), arrives at the input of 14-bit ADC (USB-3000). The absolute time reference and highly stable ADC sampling frequency necessary to detect the radio signal phase and to start data collection in accordance with the operation mode of RSDN-20 were organized using GPS Disciplined Clock (Trimble Thunderbolt E). The accuracy of pulse per second (PPS) is $\mathrm{UTC} \pm 50 \mathrm{~ns}$. The relative frequency instability of the Trimble Thunderbolt $\mathrm{E}$ harmonic generator $\Delta f / f$ after 1day operation is at least $1.16 \cdot 10^{-12}$ (a frequency difference $\Delta f$ within $3 \sigma$, and the nominal frequency $f=10$ $\mathrm{MHz}$ ). The external sampling frequency of $2.5 \mathrm{MHz}$ for ADC is obtained by separating the synchronized harmonic Trimble Thunderbolt E signal $(10 \mathrm{MHz})$. From the ADC output, the signal is fed to a laptop with 

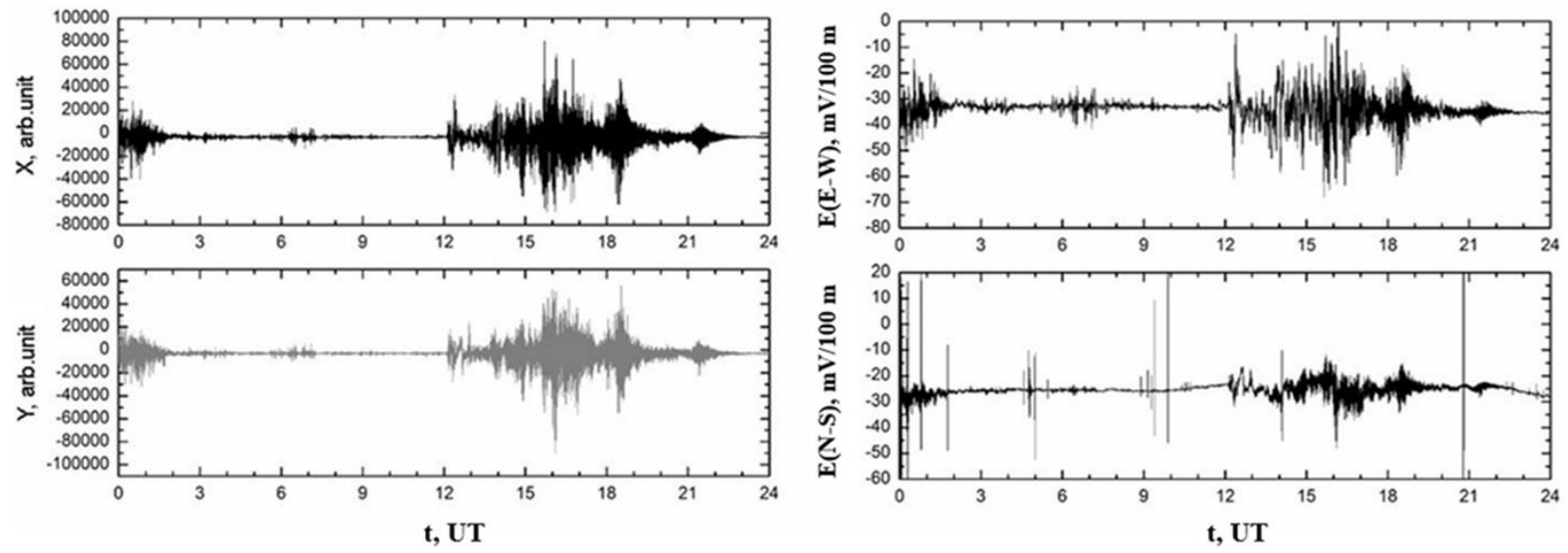

Figure 17. Variations in magnetic components and natural potentials during RC amplifications on September 8, 2017

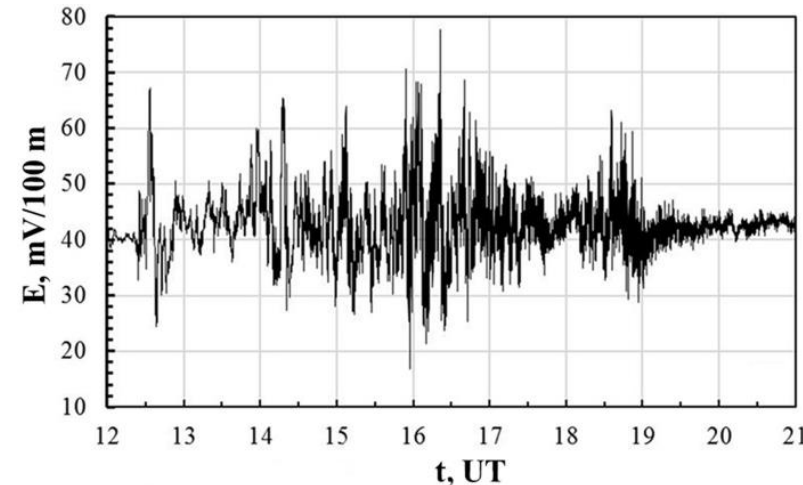

Figure 18. Variations in the $\mathbf{E}$ vector, constructed from $\mathrm{N}-$ $\mathrm{S}$ and $\mathrm{E}-\mathrm{W}$ components, during the second $\mathrm{RC}$ amplification on September 8, 2017

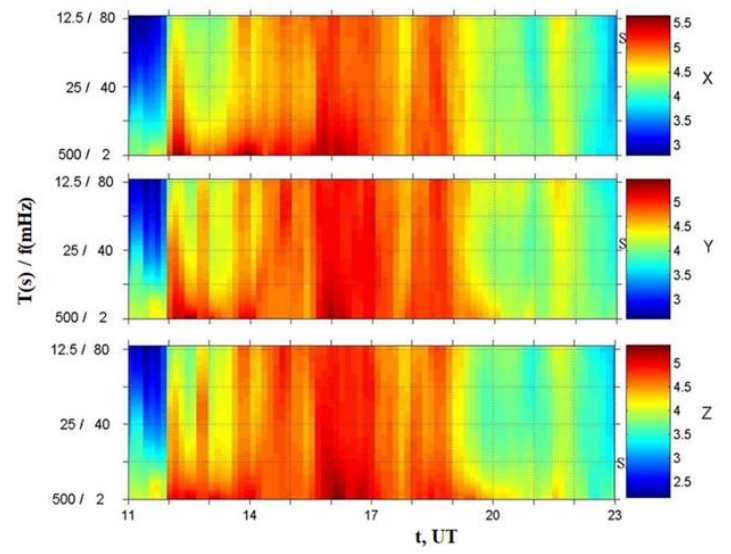

Figure 19. Spectrograms of variations in magnetic components during RC amplifications on September 8, 2017. The time is in hours from the beginning of the day

a special recording program. The gating duration of $2.688 \mathrm{~ms}$ determines the frequency resolution of the fast Fourier transform (FFT) of $372 \mathrm{~Hz}$ and corresponds to frequency multiplicity of the radio signals of interest. Before each program run, an array of trigonometric functions for FFT is formed, thus greatly speeding up the calculation of the amplitude and phase for the three frequencies. Note that the recorder of VLF radio signals has a minimum set of analog functions. The recording is based on full-scale production modules, which enables

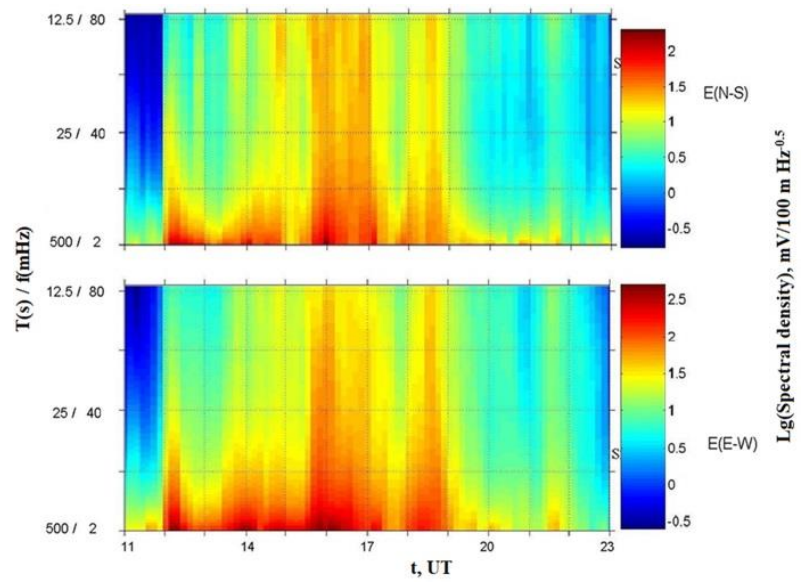

Figure 20. Spectrograms of variations in the $\mathrm{N}-\mathrm{S}$ and $\mathrm{E}-\mathrm{W}$ components of the $\mathbf{E}$ vector during the second RC amplification on September 8, 2017. The time is in hours from the beginning of the day

us to form a measurement system and eases its adjustment.

A similar recording has been made at PGO Tixie Bay since 2015. This has significantly improved the efficiency of radiophysical monitoring.

\section{EFFECTS OF SOLAR FLARES IN AMPLITUDE AND PHASE VARIATIONS OF SIGNALS FROM VLF RADIO STATIONS}

VLF radio signals can propagate over long distances in the Earth-ionosphere waveguide. An increase in the $\mathrm{X}$-ray flux during solar flares leads to a sharp increase in the electron density in the ionosphere, especially in its lower layer (D-region, 60-80 km). Such sudden changes cause sudden phase (SPA) and amplitude (SAA) anomalies in propagation of VLF electromagnetic signals [Mitra, 1977].

Propagation paths of signals from different radio stations recorded in Yakutsk and PGO Tixie Bay are shown in Figure 21.

Amplitude and phase variations in the signals from the radio station Novosibirsk at $11.904 \mathrm{kHz}$, when 


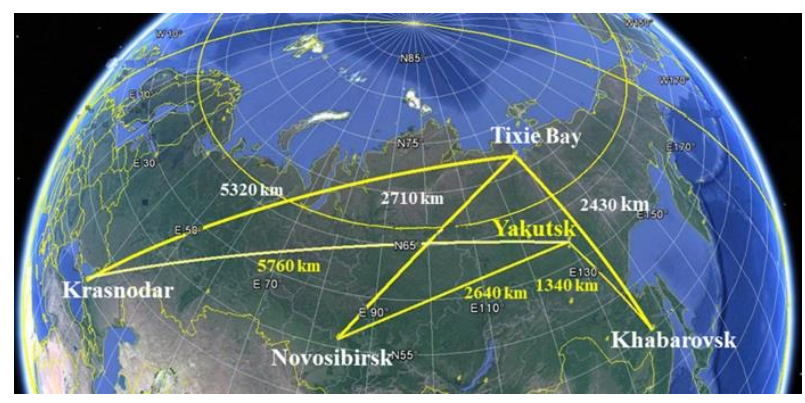

Figure 21. Propagation paths of VLF signals from radio navigation stations

recorded by PGO Tixie Bay and in Yakutsk, as well as the solar X-ray intensity and the Dst index for September 4-11, 2017 are shown in Figure $22 a-c$ respectively. Diurnal amplitude and phase variations in the radio signal, recorded under undisturbed geophysical conditions, derived by averaging values from September 1 to September 3, are also shown in Figure 22, $a, b$.

The decrease in the phase delay of the recorded signals can be interpreted as a decrease in the effective height of the Earth-lower ionosphere waveguide; and

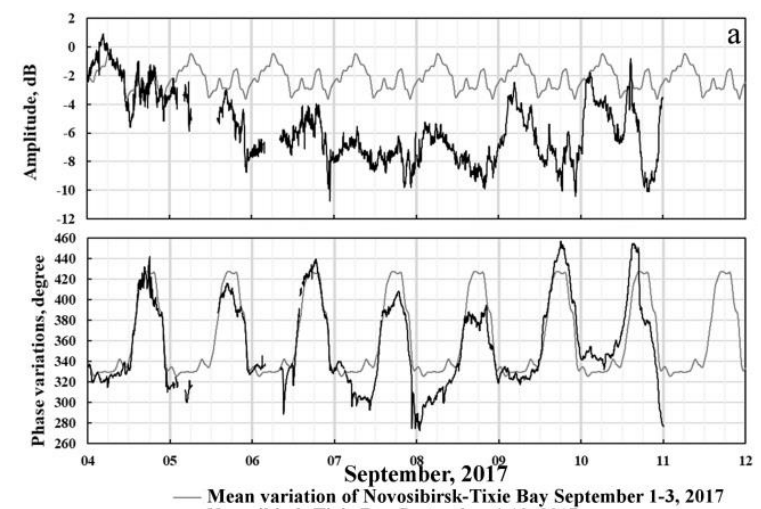

- Mean variation of Novosibirsk-Tixie Bay September 1-3, 2017
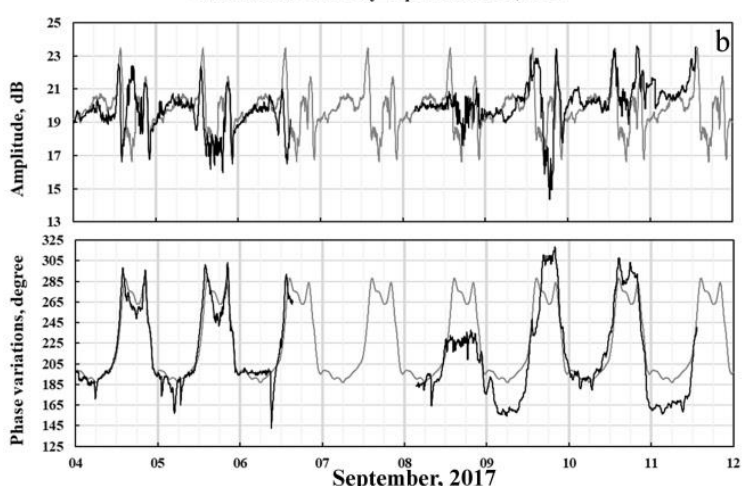

— Nean variation of Novosibirsk-Yakutsk September 1-3, 2017

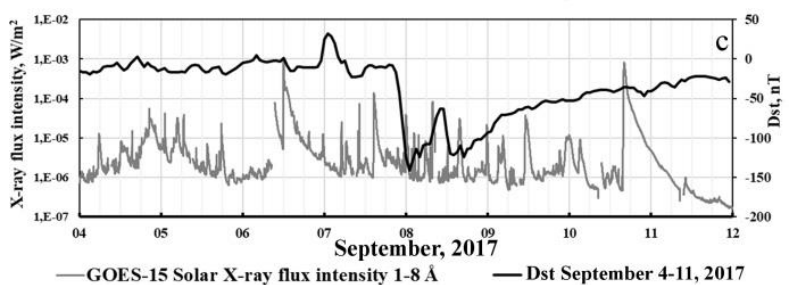

—GOES-15 Solar X-ray flux intensity 1-8 \& —Dst September 4-11, 2017

Figure 22. Variations in the amplitude and phase of signals from the radio station Novosibirsk $(11.904 \mathrm{kHz})$, recorded at PGO Tixie Bay $(a)$, and Yakutsk $(b)$, the solar X-ray flux intensity and Dst (c) on September 4-11, 2017 the lowering of the amplitude (an increase in the attenuation of radio waves during their propagation in the Earth-ionosphere waveguide), as a lowering in the vertical gradient of the electron density in the lower ionosphere [Kumar, Kumar, 2018]. Precipitation of energetic particles causes an increase in the electron density in the lower ionosphere, changing VLF radio wave propagation conditions. A peculiarity of the highlatitude ionosphere is its dynamism more largely due to precipitation of corpuscular fluxes than due to variations in solar radiation and GCR - major sources of ionization in the mid- and low-latitude ionosphere [Beloglazov, Remenets, 1982].

The radio signals recorded at Tixie Bay allow us to study the dynamics of the high-latitude ionosphere. From September 5 to September 9, there was an increase in the attenuation and a decrease in the phase delay of radio signals recorded at Tixie Bay. Hence, the maximum decrease in the amplitude of "Novosibirsk" signals on September 8 was $5 \mathrm{~dB}$; and a decrease in the phase delay was $45^{\circ}$ during the day and $55^{\circ}$ at night. Also noticeable is a decrease in the phase delay by $75^{\circ}$ on September 10, 2017 at night. Such changes in the parameters of VLF radio signals characterize the appearance of an additional ionization source - precipitation of energetic protons to the high-latitude ionosphere [Beloglazov, Remenets, 1982; Silber, Price, 2017]. The indicated time intervals coincide with an increase in integral proton fluxes (Figure 4).

Some of the electrons of internal $L$-shells (McIlwain $L$-parameter) during a magnetic storm due to pitchangle diffusion enter the loss cone, thereby causing precipitation from Earth's radiation belts into the ionosphere already at $50^{\circ}-72^{\circ}$ geomagnetic latitudes. Variations in VLF signal parameters are highly sensitive to such events [Rodger et al., 2012]. The effect of the magnetic storm revealed itself on September 8 in amplitude and phase variations of the VLF signals recorded at Yakutsk. The decrease in the phase delay on September 8 at night was $50^{\circ}$. When $D s t=-120 \mathrm{nT}$, and in daytime conditions on September 9, it was $30^{\circ}$.

The $30^{\circ}$ decrease in the phase delay of the signal "Novosibirsk" recorded at Yakutsk in daytime conditions on September 11 was caused by a sharp increase in energetic proton fluxes on September 10-11 (Figure 4). The proton event began at 17-18 UT on September 10, under night conditions along the paths considered; therefore, it manifested itself only on the next day, and from the middle of September 11 the transmitters stopped working.

Effects of X-ray flares appeared in amplitude and phase variations of radio signals during those periods when VLF propagation paths were on the sunlit side. The most sensitive parameter of VLF radio signal to geophysical events is the signal phase. For the VLF paths, this has been demonstrated by Karimov et al. [2008]. The sudden phase anomalies of VLF signals $(11.904 \mathrm{kHz})$ recorded at Tixie Bay and Yakutsk on September 6, 2017 are shown in Figure 23.

As has been mentioned above, a series of solar X-ray flares occurred from September 4 to September 10. Information about them and about SPA of signals from the radio stations at $11.904 \mathrm{kHz}$ is presented in Table 2 . 
Solar X-ray flares on September 4-10, 2017

and sudden phase anomalies of radio signals at $11.904 \mathrm{kHz}$

\begin{tabular}{|c|c|c|c|c|c|c|c|c|}
\hline \multirow{2}{*}{$\begin{array}{c}\text { Date, } \\
\text { September } \\
2017\end{array}$} & \multirow{2}{*}{$\begin{array}{l}\text { Time of the } \\
\text { maximum } \\
\text { X-ray flux }\end{array}$} & \multirow[t]{2}{*}{$\begin{array}{l}\text { Class of } \\
\text { flare }\end{array}$} & \multicolumn{2}{|c|}{$\begin{array}{c}\text { Khabarovsk } \\
\text { SPA, degree/Mm }\end{array}$} & \multicolumn{2}{|c|}{$\begin{array}{c}\text { Novosibirsk } \\
\text { SPA, degree/Mm }\end{array}$} & \multicolumn{2}{|c|}{$\begin{array}{c}\text { Krasnodar } \\
\text { SPA, degree/Mm }\end{array}$} \\
\hline & & & Yakutsk & Tixie Bay & Yakutsk & Tixie Bay & Yakutsk & Tixie Bay \\
\hline 04 & $05: 49$ & M1.2 & 9.29 & 5.35 & 6.44 & 3.32 & 4.86 & 4.70 \\
\hline 04 & $20: 33$ & M5.5 & night & night & night & night & night & night \\
\hline 04 & $22: 14$ & M2.1 & 3.57 & 3.7 & night & 0 & night & night \\
\hline 05 & $00: 35$ & C9.8 & 4.29 & 2.06 & 1.89 & 0 & night & night \\
\hline 05 & 01:08 & M4.2 & 6.43 & 8.23 & 7.20 & 2.21 & night & night \\
\hline 05 & 03:51 & M1.0 & 5 & no data & 2.65 & no data & 0 & no data \\
\hline 05 & $04: 53$ & M3.2 & 8.57 & 7.0 & 9.09 & no data & 7.12 & 0 \\
\hline 05 & $06: 40$ & M3.8 & 10 & no data & 10.61 & no data & 10.42 & no data \\
\hline 06 & $07: 34$ & C2.7 & 1.43 & no data & 0 & no data & 0 & no data \\
\hline 06 & 09:10 & $\mathrm{X} 2.2$ & 11.43 & 7.0 & 21.59 & 15.87 & 26.04 & 17.86 \\
\hline 06 & $12: 02$ & X9.3 & night & night & 3.79 & 7.38 & 22.22 & 19.74 \\
\hline 06 & $23: 39$ & M1.2 & no data & 4.94 & no data & 0 & no data & night \\
\hline 07 & 05:02 & M2.4 & no data & 10.7 & no data & 8.86 & no data & 7.71 \\
\hline 07 & $06: 28$ & C8.2 & no data & 7.0 & no data & 4.80 & no data & 0 \\
\hline 07 & 09:54 & M1.4 & no data & 0 & no data & 0 & no data & 5.45 \\
\hline 07 & $10: 15$ & M7.3 & no data & night & no data & 4.43 & no data & 9.96 \\
\hline 07 & $14: 36$ & $\mathrm{X} 1.3$ & no data & night & no data & night & no data & 0 \\
\hline 07 & 23:00 & $\mathrm{C} 2.7$ & no data & 0 & no data & 0 & no data & night \\
\hline 07 & $23: 59$ & M3.9 & no data & 7.41 & no data & 5.54 & no data & 6.02 \\
\hline 08 & $02: 24$ & M1.3 & no data & 4.53 & no data & 0 & no data & 0 \\
\hline 08 & 03:43 & M1.2 & 8.57 & 4.12 & 1.89 & 0 & 3.65 & 0 \\
\hline 08 & $05: 48$ & C8.3 & 9.30 & 3.29 & 3.03 & 2.95 & 3.99 & 0 \\
\hline 08 & 07:49 & M8.1 & 12.14 & 7.0 & 10.98 & 6.27 & 10.07 & 0 \\
\hline 08 & $12: 13$ & C5.9 & night & night & night & 0 & 6.25 & 0 \\
\hline 08 & $23: 45$ & M2.1 & 12.86 & 7.0 & 0 & 5.17 & night & night \\
\hline 09 & 03:09 & C6.3 & 5.71 & 4.53 & 0 & 3.32 & 0 & 0 \\
\hline 09 & 04:01 & C4.2 & 0 & 0 & 0 & 2.58 & 0 & 0 \\
\hline 09 & 04:28 & M1.1 & 7.14 & 5.35 & 0 & 2.58 & 0 & 0 \\
\hline 09 & 11:04 & M3.7 & night & night & 0 & 0 & 6.42 & 7.14 \\
\hline 09 & $23: 53$ & M1.1 & 2.86 & 6.17 & 0 & 0 & night & night \\
\hline 10 & 03:09 & C9.0 & 10 & 7.82 & 3.79 & 0 & 0 & 0 \\
\hline 10 & $16: 06$ & X8.2 & night & night & night & night & night & night \\
\hline
\end{tabular}

The SPA values are reduced to the unit of radio path length $(1 \mathrm{Mm})$. The lengths of the Krasnodar-Yakutsk, Novosibirsk-Yakutsk, and Khabarovsk-Yakutsk radio paths are 5.76, 2.64, and $1.34 \mathrm{Mm}$ respectively. The lengths of the Krasnodar-Tixie Bay, Novosibirsk-Tixie Bay, and Khabarovsk-Tixie Bay radio paths are 5.32, 2.71, and $2.43 \mathrm{Mm}$.

To describe SPA as a function of solar X-ray flux and zenith angle, we have used an empirical model expression [Orlov et al., 1998]

$\Phi=A+B \log (P \cos X)$,

where $\Phi$ is the change in the signal phase relative to the undisturbed level, reduced to the unit of radio path length (degree/Mm); $P$ is the $\mathrm{X}$-ray flux in the range 1 $8 \AA ; \cos X$ is the cosine of the solar zenith angle, averaged along the signal propagation path.

The selected SPA events from September 4 to September 10, when VLF signals were recorded at
Yakutsk and Tixie Bay during daytime propagation (Table 2) by the least square technique, we estimated parameters of model (1).

Solar zenith angle values along the signal propagation paths from coordinates with a resolution of $200 \mathrm{~km}$ were calculated by the algorithm [http://stjarnhi-mlen.se/comp/tutorial.html]. For the Novosibirsk-Tixie Bay and Novosibirsk-Yakutsk signal $(11.904 \mathrm{kHz})$ propagation paths, we computed SPA dependences on the solar X-ray flux. These dependences are shown in Figure 24, and their parameters for all paths of interest are listed in Table 3.

The reliability level of the parameters, calculated by the Fisher criterion, is not lower than $99 \%$.

Thus, from September 4 to September 10, 2017, in the amplitude and phase variations of radio signals we found the effects associated with X-ray solar flares, magnetic storm, and energetic proton precipitation. We established 

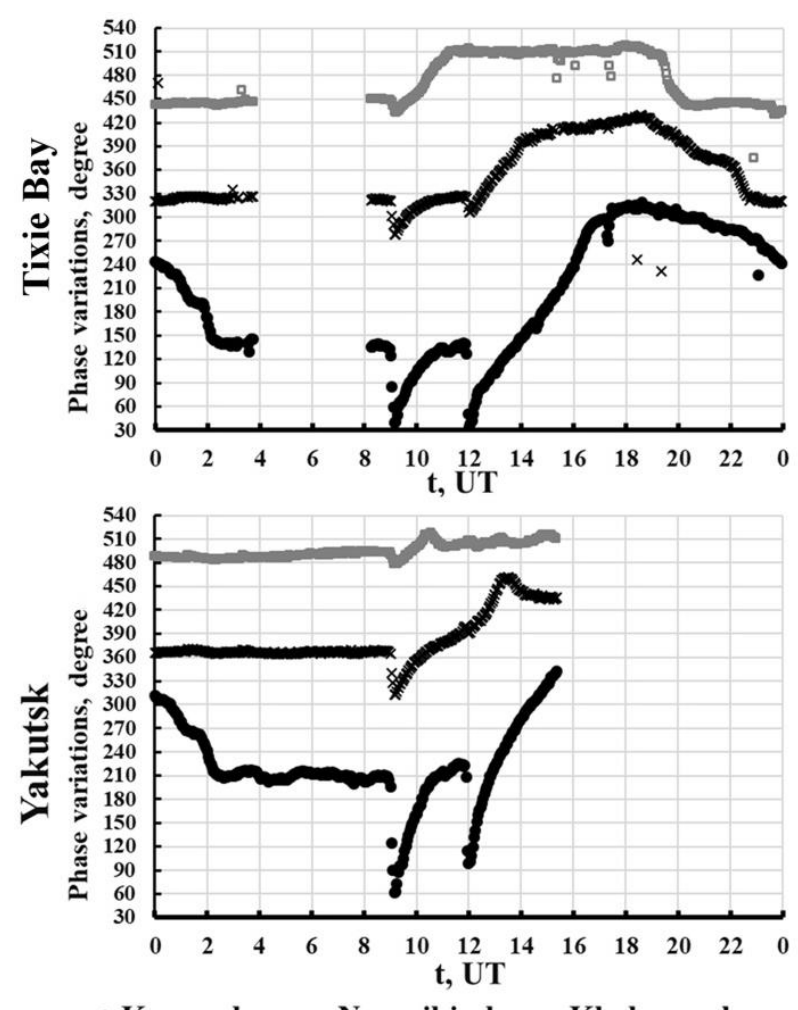

- Krasnodar × Novosibirsk "Khabarovsk

Figure 23. Sudden phase anomalies of VLF signals $(11.904 \mathrm{kHz})$, recorded at Tixie Bay and Yakutsk on September 6, 2017

that the SPA caused by the X-ray flares can be satisfactorily described by the linear function of the logarithm of the product of X-ray flux and solar zenith angle cosine averaged along a radio path. For the sixth radio paths of the VLF radio stations, we adapted parameters of this dependence, which can then be used to estimate the X-ray flux during a solar flare by recording radio signal phase variations.

In the events under study, the magnetic storm effects appeared as an increase in the attenuation and a decrease in the phase delay of the VLF radio signals recorded in the nighttime, and a daytime decrease in the phase delay along

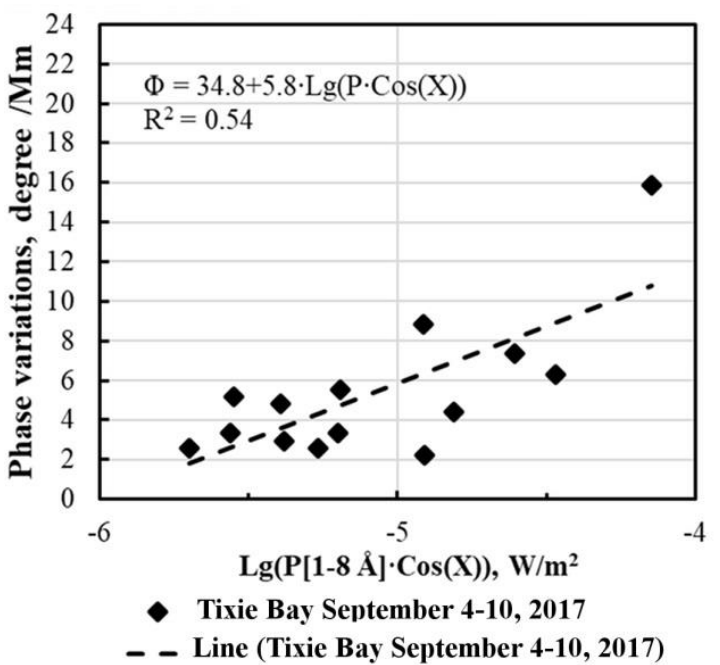

the mid-latitude radio path during the recovery of the geomagnetic field.

Additional ionization of the lower ionosphere as affected by energetic proton precipitation manifested itself as an increase in the attenuation and a decrease in the phase delay of VLF radio signals in high-latitude propagation (nighttime and daytime conditions). The increase in high-energy proton fluxes on September 10-11 also showed up as a decrease in the phase delay of VLF signals during daytime propagation in middle latitudes.

\section{CONCLUSIONS}

Thus, the comprehensive analysis leads to the following conclusions.

1. Using measurements made by GOES-15 and the worldwide network of neutron monitors, in the Ground Level Enhancement of CR (GLE72) in the energy range from $5 \mathrm{MeV}$ to $5 \mathrm{GeV}$ we have found a SCR energy spectrum, which is described by the function $J(E)=J_{0} E$ $\gamma_{\exp }\left(E / E_{0}\right)$. In particular, at 18:00 UT on September 10, 2017 , it is satisfactorily described by the approximating function $J=3027 E^{-1.99} \exp (-E / 729 \mathrm{MeV})$ (Figure 8), which is consistent with the observed generallyaccepted SCR spectrum. Using 1-hr measurements from the worldwide network of neutron monitors and the global survey method at 22:00 UT on September 6, 2017, we have made the forecast for the geomagnetic storm with $D s t<-50 \mathrm{nT}$. The forecast lead time was about a day, and at the end of September 7, 2017 the worldwide network of magnetic stations recorded a storm with $D s t=-124$ nT (Figure 5).

2. The detailed analysis of daily spectra of geomagnetic field full vector fluctuations at the observatory Yakutsk shows that their most pronounced dynamic changes are largely determined by interplanetary medium conditions (space weather) in Earth's orbit. To establish the nature of spectra of geomagnetic field fluctuations requires further systematic studies involving direct measurements of medium parameters made by spacecraft and geostationary satellites.

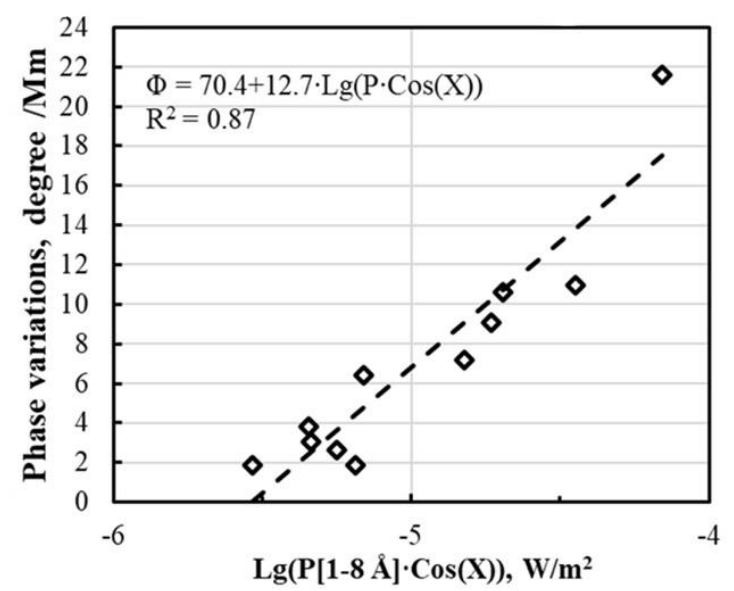

○ Yakutsk September 4-10, 2017

_ - Line(Yakutsk September 4-10, 2017)

Figure 24. Sudden phase anomalies of the signal "Novosibirsk" $(11.904 \mathrm{kHz})$ when recorded at Tixie Bay and Yakutsk as a function of solar X-ray flux 
Table 3

Parameters of SPA dependence on the logarithm of the product of X-ray flux (1-8 $\AA$ ) and the solar zenith angle cosine averaged along a propagation path

\begin{tabular}{|c|c|c|c|c|c|}
\hline Radio path & $\begin{array}{c}\text { Amount } \\
\text { of sampling }\end{array}$ & Coefficient $A$ & Coefficient $B$ & $\begin{array}{c}\text { Coefficient } \\
\text { of determination } \\
R^{2}\end{array}$ & $\begin{array}{c}\text { Standard deviation } \\
\text { of residuals, } \\
\text { degree/Mm }\end{array}$ \\
\hline $\begin{array}{c}\text { Khabarovsk-Yakutsk, } \\
1.34 \text { Mm }\end{array}$ & 17 & $38.1 \pm 9.8$ & $5.9 \pm 1.9$ & 0.36 & 2.70 \\
\hline $\begin{array}{c}\text { Khabarovsk-Tixie Bay, } \\
2.43 \mathrm{Mm}\end{array}$ & 20 & $29.5 \pm 5.9$ & $4.6 \pm 1.1$ & 0.48 & 1.67 \\
\hline $\begin{array}{c}\text { Novosibirsk-Yakutsk, } \\
2.64 \text { Mm }\end{array}$ & 11 & $70.4 \pm 8.2$ & $12.7 \pm 1.6$ & 0.87 & 2.24 \\
\hline $\begin{array}{c}\text { Novosibirsk-Tixie Bay, } \\
2.71 \text { Mm }\end{array}$ & 14 & $34.8 \pm 7.9$ & $5.8 \pm 1.6$ & 0.54 & 2.55 \\
\hline $\begin{array}{c}\text { Krasnodar-Yakutsk, } \\
5.76 \text { Mm }\end{array}$ & 10 & $56.7 \pm 10.3$ & $9.6 \pm 2.1$ & 0.72 & 4.34 \\
\hline $\begin{array}{c}\text { Krasnodar-Tixie Bay, } \\
5.32 \text { Mm }\end{array}$ & 7 & $53.3 \pm 3.3$ & $9.3 \pm 0.7$ & 0.97 & 1.1 \\
\hline
\end{tabular}

3. We have established that on September 7, 2017 at $\Phi^{\prime} \approx 62-64^{\circ} \mathrm{N}$ between Yakutsk and Tixie Bay there was an intense equivalent 2-vortex current system with auroral eastward electrojet in the late dusk sector (16:00 22:00 MLT) and westward electrojet in the late dawn sector (05:00-08:00 MLT); on September 8, 2017 there were effects of westward currents in the pre-noon sector (08:00-10:45 MLT) (continuation of the westward electrojet of the previous day) and in the midnight and early dawn sector (20:00-06:00 MLT). It is likely that the electrojet recorded in the second sector is strengthened and moved toward midnight hours, as well as to the south by the westward electrojet of the preceding day. This electrojet was located to the south of Zhigansk, but to the north of Yakutsk approximately at $\Phi^{\prime} \approx 59^{\circ} \mathrm{N}$.

4. It has been shown that during the September 8, 2017 magnetic storm from 12 to $20 \mathrm{UT}$ in a wide range of periods there were irregular pulsations - from Pi3 to Pi1. They were accompanied by variations in natural potentials of electrotelluric and geomagnetic fields with the correlation coefficient between them $\rho(E, H)=0.5 \div 0.9$ at the significance level $\alpha<0.005$.

5. The detection of signals from VLF radio stations has shown that the effects of the magnetic storms manifested themselves as an increase in the attenuation and a decrease in the phase delay of VLF radio signals at night. During the recovery of the geomagnetic field, we recorded a daytime phase delay decrease along the mid-latitude radio propagation path. Additional ionization of the lower ionosphere as affected by energetic proton precipitation revealed itself as an increase in the attenuation and a decrease in the phase delay of VLF radio signals in highlatitude propagation (nighttime and daytime). The increase in high-energy proton fluxes on September 10-11, 2017 appeared as a decrease in the phase delay of recorded VLF radio signals in daytime propagation in middle latitudes.

6. We have found that for six VLF radio paths the sudden phase anomalies caused by X-ray flares are satisfactorily described by the linear function of the logarithm of the product of X-ray flux and solar zenith angle cosine, averaged along a radio path. The model can subsequently be used to estimate the X-ray flux by recording radio signal phase variations.
This work was supported by RFBR grants No. 1842-140002-r_a, 18-45-140037-r_a, and 18-45-140028r_a, as well as by budgetary themes No. AAAA-A17117021450059-3 and AAAA-A17-117021450058-6.

\section{REFERENCES}

Aleksandrov M.S., Bakleneva Z.M., Gladshtein N.D., Ozerov V.P., Potapov A.V., Remizov L.T. Fluktuatsii elektromagnitnogo polya Zemli $v$ diapazone SNCh [Fluctuations of the Earth's electromagnetic field in the SLF range]. Moscow, Nauka Publ., 1972, 195 p. (In Russian).

Baishev D.G., Moiseev A.V., Boroev R.N., Makarov G.A., Poddel'skiy I.N., Poddel'skiy A.I., Shevtsov B.M., Yumoto K. International MAGDAS Project: first results of geomagnetic observations in Yakutia. Nauka i obrazovanie [Science and Education]. 2013, no. 1 (69), pp. 7-10. (In Russian).

Beloglazov M.I., Remenets G.F. Rasprostranenie sverkhdlinnykh radiovoln $v$ vysokikh shirotakh [Superlong radio waves propagation in high latitudes]. Leningrad, Nauka Publ., 1982, 237 p. (In Russian).

Berezhko E.G., Taneev S.N. Shock acceleration of solar cosmic rays. Astron. Lett. 2013, vol. 39, no. 6, pp. 393-403. DOI: 10.1134/S1063773713060017.

Blackman R.B. The Measurement of Power Spectra from the Point of View of Communications Engineering. New York, Dover Publ., 1958, 120 p.

Cheng D.Y. Anomalous short-period pulsations in GOES magnetometer data before solar proton events. Solar Phys. 1991, vol. 131, pp. 395-406.

Ellison D.C., Ramaty R. Shock acceleration of electrons and ions in solar flares. Astrophys. J. 1985, vol. 298, pp. 400-408.

Hessler V.P., Wescott E.M. Correlation between earth current and geomagnetic disturbance. Nature. 1959, vol. 184, pp. 627.

Grigoryev V.G., Starodubtsev S.A. Global survey method in real time and space weather forecasting. Bull. RAS: Phys. 2015, vol. 79, pp. 649-653. DOI: 10.3103/S1062873815050226.

Grigoryev V.G., Starodubtsev S.A., Potapova V.D. Definition of parameters of daily anisotropy of cosmic rays according to the world network of neutron monitors. $J$. Phys.: Conf. Ser. 2013, vol. 409, iss. 1, article id. 012172. DOI: 10.1088/1742-6596/409/1/012172.

Grigoryev V.G., Starodubtsev S.A., Gololobov P.Y. Monitoring of geomagnetic disturbance predictors from cosmic ray ground measurements. Bull. RAS: Phys. 2017, vol. 81, no. 2, pp. 200-202.

Karimov R.R., Kozlov V.I., Mullayarov V.A. Specific features of variations in the characteristics of VLF signals 
when the lunar shadow propagated along the path during the solar eclipse of March 29, 2006. Geomagnetism and Aeronomy. 2008, vol. 48, no. 2, pp. 240-244.

Karimov R.R., Kozlov V.I., Korsakov A.A., Mullayarov V.A., Mel'chinov V.P. Variations of very low frequency signal parameters of radio navigation stations registered in Yakutsk. Sovremennye problemy distantsionnogo zondirovaniya Zemli $i z$ kosmosa [Current problems in remote sensing of the Earth from space]. 2012, vol. 9, no. 4, pp. 57-62. (In Russian).

Kleymenova N.G. Geomagnetic pulsations. Model' kosmosa. Kn. 1: Fizicheskie usloviya $v$ kosmicheskom prostranstve [Space Model. Vol. 1: Physical Conditions in Space]. Moscow, MGU Publ., 2007, vol. 1, pp. 611-626. (In Russian).

Kobrin M.M., Malygin V.I., Snegirev S.D. Long-period pulsations of the Earth's magnetic field with periods more than 20 minutes before proton flares on the Sun. Planet. Space Sci. 1985, vol. 33, pp. 1251-1257.

Krolevets A.N., Kopylova G.N. Tidal components in the electrotelluric field. Izvestiya. Physics of the Solid Earth. 2003, vol. 39, no. 5, pp. 418-427.

Krymsky G.F., Grigor'ev V.G., Starodubtsev S.A. New method for estimating the absolute flux and energy spectrum of solar cosmic rays based on neutron-monitor data. JETP Lett. 2008, vol. 99, no. 7, pp. 411-413.

Krymsky G.F., Grigoryev V.G., Starodubtsev S.A., Taneev S.N. Ground-level enhancement of solar cosmic ray on October 28, 2003: a mechanism of the generation of particles in the Sun. JETP Lett. 2015, vol. 102, pp. 335-342.

Kumar A., Kumar S. Solar flare effects on D-region ionosphere using VLF measurements during low- and highsolar activity phases of solar cycle 24. Earth, Planets and Space. 2018, 70:29. DOI: 10.1186/s40623-018-0794-8.

Lovell J.L., Duldig M.L., Humble J.E. An extended analysis of the September 1989 cosmic ray ground level enhancement. $J$. Geophys. Res.: Space Phys. 1998, vol. 103, pp. 23733-23742.

Mitra A.P. Vozdeistvie solnechnykh vspyshek na ionosferu Zemli [Effects of solar flares on Earth's ionosphere]. Moscow, Mir Publ., 1977. 370 p. English edition: Mitra A.P. Ionospheric Effects of Solar Flares. Dordrecht, Holland, D. Reidel Publ. Co., 1974. 307 p.

Moiseev A.V., Makarov G.A., Neustroev N.I. Geomagnetic investigations in the north-eastern Russia. Vestnik Otdeleniya nauk o Zemle RAN [Bull. Department of Earth Sciences RAS] 2011, NZ5004. DOI: 10.2205/2011NZ000106. (In Russian).

Nymmik R.A. Inflections (knees) in wide-range spectra of solar energetic protons and heavy ions: their form, parameters, and regularities. Bull. RAS: Phys. 2011, vol. 75, no. 6, pp. 761-763. DOI: 10.3103/S1062873811060335.

Orlov A.B., Pronin A.E., Uvarov A.N. Latitudinal dependence of the effective electron-loss coefficient in the daytime lower ionosphere as deduced from VLF phase variations and riometric absorption data during SIDs. Geomagnetism and Aeronomy. 1998, vol. 38, no. 3, pp. 341-346.

Plotnikov I.Ya., Shadrina L.P., Starodubtsev S.A., Krymsky G.F. Coronal mass ejection, geomagnetic storms and ground-based cosmic ray intensity decreases. Proc. $10^{\text {th }}$ Int. Conf. "Problems of Geocosmos". St. Petersburg, Petrodvorets, Russia, October 2014, pp. 351-354.

Rodger C.J., Clilverd M.A., Kavanagh A.J., Watt C.E.J., Verronen P.T., Raita T. Contrasting the responses of three different ground-based instruments to energetic electron precipitation. Radio Sci. 2012, vol. 47, no. 2, RS20211-13. DOI: $10.1029 / 2011$ RS004971.

Shadrina L.P., Starodubtsev S.A. Manifestation of interplanetary shock in geomagnetic storms and substorms. Physics of Auroral Phenomena. 2016a, vol. 39, pp. 23-26.

Shadrina L.P., Starodubtsev S.A. Effect of IMF turbulence in the vicinity of interplanetary shocks on geomagnetic storms and substorms generation. Proc. $11^{\text {th }}$ Int. Conf. "Problems of
Geocosmos", St. Petersburg, Petrodvorets, Russia, October, 2016, 2016b, pp. 103.

Shadrina L.P., Barkova E.S., Plotnikov I.Ya., Starodubtsev S.A. Large-scale solar wind disturbances as a reason of intense geomagnetic storms. Proc. $9^{\text {th }}$ Int. Conf. "Problems of Geocosmos". St. Petersburg, Petrodvorets, Russia, October 2012, pp. 381-386.

Shadrina L.P., Krymsky G.F., Plotnikov I.Ya., Starodubtsev S.A. Interplanetary shock geoeffectivity during the growth phase of solar activity. Proc. $10^{\text {th }}$ Int. Conf. "Problems of Geocosmos". St. Petersburg, Petrodvorets, Russia, October 2014, pp. 388-391.

Silber I., Price C. On the use of VLF narrowband measurements to study the lower ionosphere and the mesosphere - lower thermosphere. Surveys in Geophysics, 2017, vol. 38, pp. 407-441.

Starodubtsev S.A., Grigor'ev V.G., Gololobov P.Yu. Kuzmin A.I. Yakutsk Cosmic Ray Spectrograph. Sbornik trudov Vserossiiskoi konferentsii "Geliogeofizicheskie issledovaniya $v$ Arktike" [Proc. National Conference "Heliogeophysical Research in Arctic"]. Moscow, 2016, pp. 125-128. (In Russian).

Starodubtsev S.A., Grigoryev V.G., Gololobov P.Y. The A.I. Kuz'min cosmic ray spectrograph: New scintillation muon telescopes. Bull. RAS: Phys. 2017, vol. 81, iss. 4, pp. 538-541.

URL: ftp://ftp.swpc.noaa.gov/pub/lists/pchan/README (accessed October 9, 2018).

URL: http://stjarnhi-mlen.se/comp/tutorial.html (accessed October 9, 2018).

URL: $\quad$ https://satdat.ngdc.noaa.gov/sem/goes/data/new avg/2017/09/goes15/csv/g15_xrs_1m_20170901_20170930.csv

(accessed October 9, 2018). 2018)

URL: http://www.spaceweather.com (accessed October 9,

URL: https://en.wikipedia.org/wiki/Geomagnetic_storm (accessed October 9, 2018).

URL: https://satdat.ngdc.noaa.gov/sem/goes/data/new_ avg/2017/09/goes15/csv (accessed October 9, 2018).

URL: http://www.solarham.net/top10.txt (accessed October 9, 2018).

URL: https://www.spaceweatherlive.com (accessed October 9, 2018).

URL: http://www.solarham.net (accessed October 9, 2018).

URL: http://wdc.kugi.kyoto-u.ac.jp/dst_realtime/index.html (accessed October 9, 2018).

URL: http://www.ysn.ru/ipm (accessed October 9, 2018).

URL: http://www.ysn.ru/smt (accessed October 9, 2018).

URL: ftp://ftp.swpc.noaa.gov/pub/lists/pchan (accessed October 9, 2018).

URL: http://www.nmdb.eu (accessed October 9, 2018).

URL: http://www.intermagnet.org (accessed October 9, 2018).

URL: http://magdas2.serc.kyushu-u.ac.jp/station/index.html (accessed October 9, 2018).

URL: https://cdaweb.sci.gsfc.nasa.gov/index.html (accessed October 9, 2018).

URL: http://www.stce.be/newsletter/pdf/2017/STCEnews 20170915.pdf (accessed October 9, 2018).

URL: http://www.ysn.ru/ starodub/SpaceWeather/global_ survey_real_time.html (accessed October 9, 2018).

How to cite this article

Starodubtsev S.A., Baishev D.G., Grigoryev V.G., Karimov R.R., Kozlov V.I., Korsakov A.A., Makarov G.A., Moiseev A.V. Analyzing solar, cosmic, and geophysical events in September 2017, using SHICRA SB RAS complex observations. Solar-Terrestrial Physics. 2019. Vol. 5. Iss. 1. P. 14-27. DOI: $10.12737 /$ stp-51201903. 